\title{
Uncovering the relation of a scalar resonance to the Higgs boson
}

\author{
Adrián Carmona, ${ }^{*}$ Florian Goertz, ${ }^{\dagger}$ and Andreas Papaefstathiou ${ }^{\ddagger}$ \\ CERN, Theoretical Physics Department, CH-1211 Geneva 23, Switzerland \\ (Received 26 June 2016; revised manuscript received 27 December 2016; published 26 May 2017)

\begin{abstract}
We consider the associated production of a scalar resonance with the standard model Higgs boson. We demonstrate via a realistic phenomenological analysis that couplings of such a resonance to the Higgs boson can be constrained in a meaningful way in future runs of the LHC, providing insights on its origin and its relation to the electroweak symmetry breaking sector. Moreover, the final state can provide a direct way to determine whether the new resonance is produced predominantly in gluon fusion or quark-antiquark annihilation. The analysis focuses on a resonance coming from a scalar field with vanishing vacuum expectation value and its decay to a photon pair. It can, however, be straightforwardly generalized to other scenarios.
\end{abstract}

DOI: 10.1103/PhysRevD.95.095022

\section{NEW SCALAR RESONANCES AT COLLIDERS}

Models with an additional (pseudo)scalar singlet with a mass of several hundred $\mathrm{GeV}$ represent a well motivated class of extensions of the Standard Model (SM) of particle physics, including composite Higgs scenarios, supersymmetry, Coleman-Weinberg models, models addressing the strong $C P$ problem, models of flavor, as well as generic Higgs portal setups (see e.g. [1-9]). A particularly promising channel to search for and analyze such a particle is its decay to two photons. Beyond being possibly sizable in certain scenarios, it offers a robust and clean way to detect a signal, emerging over a steeply falling background $[10,11]$.

After its discovery, an important aspect of scrutinizing any new resonance is in fact to measure its couplings, and hence determine its relation, to the known particle content of the SM. A crucial component of this task is to uncover its role in the arena of electroweak symmetry breaking (EWSB). As a first step in this direction, determination of the couplings of the new scalar to the SM-like Higgs boson is mandatory, which is the main focus of this article, employing its diphoton $(\gamma \gamma)$ decay channel. ${ }^{1}$

\footnotetext{
${ }^{1}$ A specific motivation for the first version of this manuscript was provided by the apparent $\gamma \gamma$ resonance at $M_{\gamma \gamma} \sim 750 \mathrm{GeV}$ in ATLAS $[12,13]$ and CMS $[14,15]$ data. This turned out not to be present in the 2016 data $[16,17]$. Consequently the article was generalized to other mass scales of a potential scalar resonance, which remains well motivated, taking into account new limits on its cross section-see below. Comprehensive analyses studying constraints on (other) possible couplings of a diphoton resonance as well as detailed examinations of indirect footprints of new (high multiplicity) sectors, linked to its productions or decay appeared e.g. in [18-45].

adrian.carmona@cern.ch

†florian.goertz@cern.ch

*apapaefs@cern.ch
}

Published by the American Physical Society under the terms of the Creative Commons Attribution 3.0 License. Further distribution of this work must maintain attribution to the author(s) and the published article's title, journal citation, and DOI.
For a $\gamma \gamma$ resonance originating from a scalar field $\mathcal{S}$, neutral under the SM gauge group, the relevant effective Lagrangian for our study-augmenting the SM at dimension $D \leq 5$-is

$$
\begin{aligned}
\mathcal{L}_{\text {eff }} \supset & \frac{1}{2} \partial_{\mu} \mathcal{S} \partial^{\mu} \mathcal{S}-\frac{1}{2} \mu_{S}^{2} \mathcal{S}^{2} \\
& -\left(y_{d}^{S}\right)^{i j} \frac{\mathcal{S}}{\Lambda} \bar{Q}_{L}^{i} H d_{R}^{j}-\left(y_{u}^{S}\right)^{i j} \frac{\mathcal{S}}{\Lambda} \bar{Q}_{L}^{i} \tilde{H} u_{R}^{j}+\text { H.c. } \\
& -\frac{\mathcal{S}}{\Lambda} \frac{1}{16 \pi^{2}}\left[g^{\prime 2} c_{B}^{S} B_{\mu \nu} B^{\mu \nu}+g^{2} c_{W}^{S} W^{I \mu \nu} W_{\mu \nu}^{I}\right. \\
& \left.+g_{s}^{2} c_{G}^{S} G^{a \mu \nu} G_{\mu \nu}^{a}\right]-\lambda_{H S}|H|^{2} \mathcal{S}^{2}-\frac{\lambda_{S}}{4} \mathcal{S}^{4} .
\end{aligned}
$$

Here, $Q_{L}^{i}$ is the $i$ th generation left-handed $S U(2)_{L}$ fermion doublet, $d_{R}^{j}$ and $u_{R}^{j}$ are the right-handed $S U(2)_{L}$ fermion singlets for generation $j,\left(y_{q}^{S}\right)^{i j}$ are the corresponding Yukawa-like couplings, $c_{B}^{S}, c_{W}^{S}$ are the couplings of $\mathcal{S}$ to the $U(1)_{Y}$ and $S U(2)_{L}$ gauge fields $B$ and $W, c_{G}^{S}$ is the coupling to the gluon fields, $H$ is the Higgs boson doublet, $\lambda_{\mathrm{HS}}$ is the Higgs-scalar portal coupling, and $\lambda_{S}$ is the new scalar quartic. Moreover, $\Lambda$ denotes the scale of heavy new physics (NP), mediating the contact interactions of $\mathcal{S}$ with SM gauge bosons and fermions (the latter involving $H$ to generate a gauge singlet). Note that we do not include terms with an odd number of $\mathcal{S}$ fields containing only scalars (as well as lepton fields). The corresponding interaction vertices will turn out irrelevant in general for the process we will consider; see below. ${ }^{2}$ Beyond that, terms linear in $\mathcal{S}$ could also lead to the singlet mixing with the Higgs boson after EWSB, which would in fact affect its phenomenology. Although such effects could still be present at a nonnegligible level, they are expected to be subleading and we neglect them for simplicity; see Appendix B. Furthermore,

\footnotetext{
${ }^{2}$ The full list of potential $D \leq 5$ operators is listed in Appendix A.
} 
the analysis that follows is independent of the $C P$ properties of $\mathcal{S}$ and its interactions and henceforth, for simplicity, we assume it to be $C P$-even with $C P$-conserving interactions.

With the potential for the Higgs doublet $H$ taking the conventional form

$$
\mathcal{V}=\lambda_{H}|H|^{4}+\mu_{H}^{2}|H|^{2},
$$

and assuming that $H$ is the only scalar that gets a vacuum expectation value (VEV), $|\langle H\rangle|=v / \sqrt{2}$, triggering EWSB, we obtain the condition ${ }^{3}$

$$
\lambda_{H S} \mu_{H}^{2}-\lambda_{H} \mu_{S}^{2}<0, \quad \mu_{H}^{2}<0 .
$$

The physical mass of the singlet thus reads $M=$ $\sqrt{\mu_{S}^{2}+\lambda_{H S} v^{2}}$.

The resulting trilinear interactions between the physical scalar resonances after EWSB are described by

$$
\mathcal{L}_{\text {scalar }}^{3}=-\frac{M_{h}^{2}}{2 v} h^{3}-\lambda_{H S} v h S^{2},
$$

where $h$ is the Higgs boson, which (due to the case of negligible scalar mixing) is basically fully embedded in $H$ and describes excitations around its VEV, such that in unitary gauge $H \simeq 1 / \sqrt{2}(0, v+h)^{T}$, and $S$ is a new scalar resonance, which can also be (approximately) identified as $\mathcal{S}=S$. Moreover, $v \simeq 246 \mathrm{GeV}$ is the Higgs $\mathrm{VEV}, M_{h} \simeq$ $125 \mathrm{GeV}$ is the measured Higgs boson mass, and the portal coupling $\lambda_{H S}$ is to be determined, being a main scope of this paper. This coupling would basically be unconstrained by direct observation of a diphoton resonance. Nevertheless, loose indirect constraints can be derived, requiring vacuum stability not to be spoiled. They read

$$
\lambda_{S}>0, \quad \lambda_{H}>0, \quad \lambda_{H S}^{2}<\lambda_{H} \lambda_{S},
$$

and need to be imposed at least at the scale where the NP enters, i.e., the $\mathrm{TeV}$ scale (see e.g. $[1,46]) .{ }^{4}$ Requiring $\lambda_{H} \sim 0.13$, to fit the observed Higgs mass, as well as $\lambda_{S}<(4 \pi)^{2}$, we thus obtain $-4.5 \lesssim \lambda_{H S} \lesssim 4.5$. We will see below that in general our analysis can put stronger bounds than these on $\lambda_{H S}{ }^{5}$

\footnotetext{
${ }^{3}$ The fact that $\langle\mathcal{S}\rangle=0$ guarantees the full absence of scalar mixing, which could otherwise occur even without linear terms in $S$.

${ }^{4}$ While the first two conditions need to hold at all scales, for $\lambda_{H S}>0$ the last condition might be violated at higher scales, while the electroweak vacuum still remains stable [1]. Moreover, for the latter condition odd terms in $\mathcal{S}$ are assumed to vanish.

${ }^{5}$ Note that requiring a more conservative limit, such as $\lambda_{S}<$ $\mathcal{O}(10)$ (corresponding to e.g. $d \lambda_{S} / \lambda_{S}<1[1]$ ), restricts $\lambda_{H S}$ to be not much larger than 1 and would remove a considerable portion of the parameter space where our analysis exhibits sensitivity. However, in any case, the limits presented here are complementary to such considerations.
}

In the present study we consider measurement of the coupling $\lambda_{H S}$ at the LHC, where it can be probed via the associated production of the new resonance with the SMlike Higgs boson: $p p \rightarrow h S$. For this process, the interactions neglected in (1) play no role to good approximation: they would either not enter at leading order (LO) or, as is the case for the $|H|^{2,4} \mathcal{S}$ interactions, contribute at most to a diagram with a (strongly suppressed) off-shell Higgs boson propagator; for details see Appendixes A and B.

In principle several decay modes of $S$ can be considered. Here we focus on the process $p p \rightarrow h S \rightarrow h \gamma \gamma$, where the new particle decays to a pair of photons. Given that the tentative cross section of the resonant diphoton production, $p p \rightarrow S \rightarrow \gamma \gamma$, will be known and will be well measured in the case of discovery, this allows constraints on the coupling $\lambda_{H S}$ to be imposed almost independently of the couplings to the initial-state partons and final-state photons, given that only a single production mode is relevant. We consider production via gluon fusion and quark-antiquark annihilation, mediated through nonvanishing coefficients $c_{G}^{S},\left(y_{d}^{S}\right)^{22}$, or $\left(y_{d}^{S}\right)^{33}$, respectively, and show how these modes could be disentangled via appropriate measurements. We will implicitly assume not too large values of $\Gamma(S \rightarrow \gamma \gamma)$, in such a way that photoproduction is always subdominant.

Although we will focus on three specific benchmark masses of $M=600,750,900 \mathrm{GeV}$, our analysis could be applied to the general case of associated production of a Higgs boson with a scalar diphoton resonance of any mass. Moreover, several features of the final state studied here, such as the invariant mass of the final-state scalar or the total invariant mass of the process, will exhibit similar features when considering other decay modes.

The article is organized as follows: in Sec. II we examine the process of associated production of a scalar resonance and a Higgs boson, in Sec. III we describe the event generation and detector simulation setup, and in Sec. IV we provide details of the analysis and results. Finally, we conclude in Sec. V.

\section{ASSOCIATED PRODUCTION OF A SCALAR RESONANCE AND A HIGGS BOSON}

\section{A. Production through gluon fusion}

The dominant diagrams at LO contributing to the production of the $h S$ final state in gluon fusion and subsequent decay of the resonance $S$ to a pair of photons via the interactions

$$
\begin{aligned}
\mathcal{L}_{\text {eff }} & \supset-\frac{S}{\Lambda} \frac{1}{16 \pi^{2}}\left[e^{2}\left(c_{B}^{S}+c_{W}^{S}\right) F_{\mu \nu} F^{\mu \nu}+g_{s}^{2} c_{G}^{S} G^{a \mu \nu} G_{\mu \nu}^{a}\right] \\
& =-\frac{S}{\Lambda} \frac{1}{16 \pi^{2}}\left[e^{2} c_{\gamma}^{S} F_{\mu \nu} F^{\mu \nu}+g_{s}^{2} c_{G}^{S} G^{a \mu \nu} G_{\mu \nu}^{a}\right]
\end{aligned}
$$

are shown in Fig. 1. In the analysis of the present article, we will consider the Higgs boson decaying to a bottom-quark 


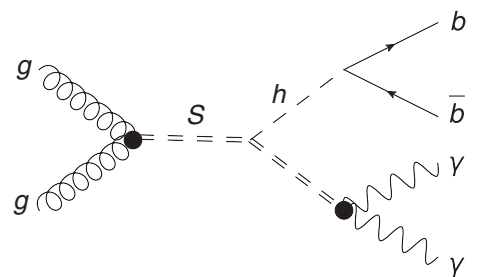

(a)

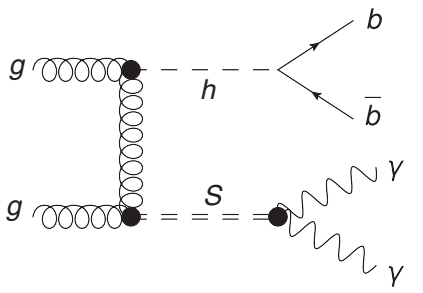

(b)

FIG. 1. The diagrams contributing to the process $g g \rightarrow h S \rightarrow$ $(b \bar{b})(\gamma \gamma)$ at the LHC at LO.

pair, since this maximizes the expected number of events, which would be modest in general. There exist both the $s$-channel $S$ exchange, involving the portal coupling $\lambda_{H S}$ and depicted in 1(a), and the "direct" $h S$ production, via $t$-channel gluon exchange, depicted in 1(b).

\section{B. Production through quark-antiquark annihilation}

For the case of quark-antiquark annihilation, a new diagram arises from the contact interaction $q \bar{q} h S .^{6}$ Both contributing graphs are shown in Fig. 2. The new diagram (b) distinguishes the $q \bar{q}$ annihilation from the gluon fusion case. An important fact is that now the $h S$ process is nonvanishing and significant even in the absence of the portal coupling $\lambda_{H S}$. This indicates that one can employ this final state to exclude $q \bar{q}$ annihilation as the dominant production process (in the absence of a signal). In the following we will focus on the cases of $q=b, q=s$, or $q=c$.

It will be useful in both scenarios to construct the ratio of the associated production process $p p \rightarrow h S$, through all possible intermediate states, with the subsequent decay of the new resonance to a $\gamma \gamma$ final state, to that of the single production $p p \rightarrow S \rightarrow \gamma \gamma$,

$$
\rho\left(x x^{\prime}\right)=\frac{\sigma\left(x x^{\prime} \rightarrow h S \rightarrow h \gamma \gamma\right)}{\sigma\left(x x^{\prime} \rightarrow S \rightarrow \gamma \gamma\right)},
$$

where we consider $x x^{\prime}=\{g g, b \bar{b}, s \bar{s}, c \bar{c}\} .^{7}$ The ratio is a useful quantity since it removes the dependence on the

\footnotetext{
${ }^{6}$ The $t$-channel diagram with the $q \bar{q} h$ interaction is suppressed due to a small Yukawa coupling.

${ }^{7}$ In the most general setup, the analysis of this article can constrain the sum of the squares of the couplings of $S$ to all quark generations (for a given $\lambda_{H S}$ ), appropriately weighted by the parton density functions.
}

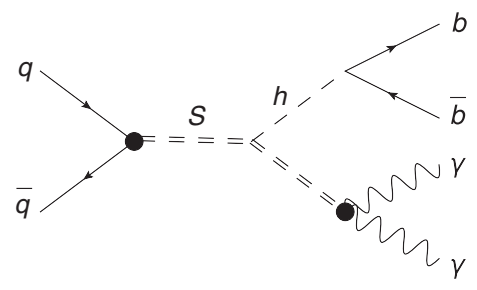

(a)

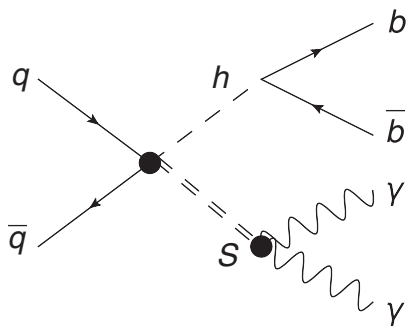

(b)

FIG. 2. The diagrams contributing to the process $q \bar{q} \rightarrow h S \rightarrow$ $(b \bar{b})(\gamma \gamma)$ at the LHC at LO.

product of couplings of the new resonance to the initialstate partons and final-state photons. Moreover, it can be used to absorb, at least approximately, theoretical and experimental systematic uncertainties. ${ }^{8}$

We show the dependence of the ratio $\rho$ on the portal coupling $\lambda_{H S}$ in Fig. 3 for $g g, b \bar{b}, s \bar{s}$, and $c \bar{c}$ initial states for the example diphoton resonance mass $M=750 \mathrm{GeV}$ and width $\Gamma=1 \mathrm{GeV}^{9}$ A width of $\Gamma \lesssim 1 \mathrm{GeV}$ can be obtained, for example, if $c_{\gamma}^{S} \sim \mathcal{O}(10)$ and $c_{G} \sim \mathcal{O}(1)$ or $\left(y_{d}^{S}\right)^{33} \sim \mathcal{O}(1)$, for $\Lambda=1 \mathrm{TeV}$, with a cross section in $p p \rightarrow S \rightarrow \gamma \gamma$ compatible with the signal assumed below. Similar behavior of the ratio $\rho$ is obtained for different scalar $S$ masses and widths.

Since the dominant matrix-element contribution to the $g g$-initiated $h S$ process is proportional to the portal coupling, the process approximately vanishes as $\lambda_{H S} \rightarrow 0$, and hence $\rho(g g) \simeq \rho_{2 g g} \lambda_{H S}^{2}$, where $\rho_{2 g g} \approx 0.00133$, for $M=$ $750 \mathrm{GeV}$ and $\Gamma=1 \mathrm{GeV}$, obtained by performing a quadratic fit of the $g g$ curve in Fig. 3. As already discussed, this does not hold for the $q \bar{q}$-initiated process due to the contact interaction diagram. This results in a non-negligible minimum for $\rho(q \bar{q})$. A fit to the cross section, again for $M=750 \mathrm{GeV}$ and $\Gamma=1 \mathrm{GeV}$, yields $\rho(q \bar{q}) \simeq \rho_{2 q \bar{q}} \lambda_{H S}^{2}+$ $\rho_{1 q \bar{q}} \lambda_{H S}+\rho_{0 q \bar{q}} \quad$ with $\rho_{0 b \bar{b}} \approx 0.00828, \quad \rho_{1 b \bar{b}} \approx 0.00309$,

\footnotetext{
${ }^{8}$ For a similar idea investigated in the context of Higgs boson pair production, see [47].

${ }^{9}$ We employ a single cut of $M_{\gamma \gamma}>200 \mathrm{GeV}$ at generation level in order to remove (SM-like) $p p \rightarrow h h \rightarrow h \gamma \gamma$ interference with the signal, i.e., $S(\gamma \gamma)+h$ production. Only after this cut can we identify a "signal" contribution to the actual physical processwhich is Higgs production in association with a photon pairunambiguously with the process $p p \rightarrow h S \rightarrow h \gamma \gamma$ to good approximation, assuming the model (1).
} 


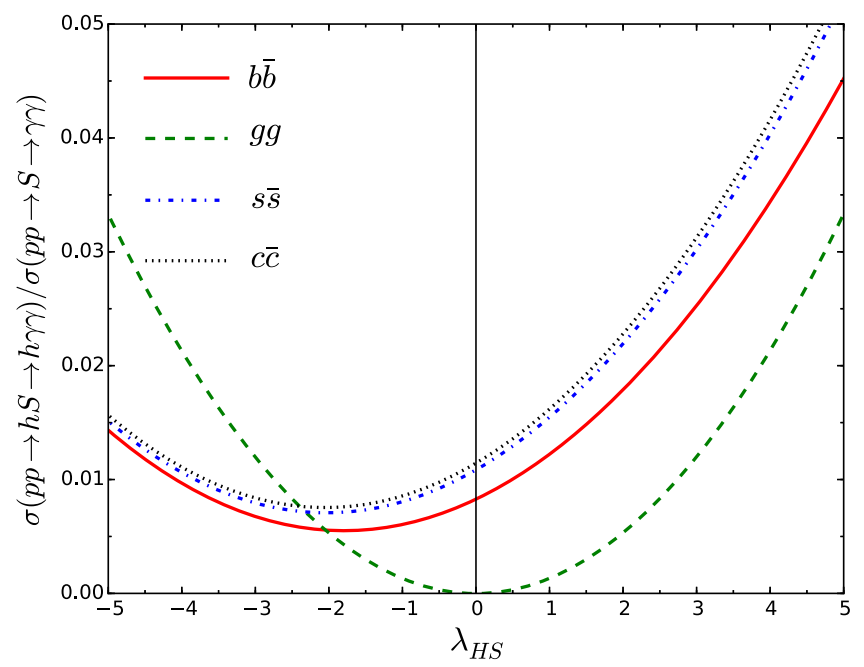

FIG. 3. The ratios $\rho(g g), \rho(b \bar{b}), \rho(s \bar{s})$, and $\rho(c \bar{c})$ for $g g, b \bar{b}, s \bar{s}$, and $c \bar{c}$ initial states, respectively, defined between the associated production $p p \rightarrow h S \rightarrow h \gamma \gamma$ and the single production $p p \rightarrow S \rightarrow \gamma \gamma$, as functions of the portal coupling $\lambda_{H S}$. The mass of the scalar resonance was taken to be $M=750 \mathrm{GeV}$ and the width $\Gamma=1 \mathrm{GeV}$.

$\rho_{2 b \bar{b}} \approx 0.00086$, corresponding to $b \bar{b}$ initial states, and $\rho_{0 s \bar{s}} \approx 0.01025, \quad \rho_{1 s \bar{s}} \approx 0.00371, \quad \rho_{2 s \bar{s}} \approx 0.00091$, corresponding to $s \bar{s}$ initial states. The case of $c \bar{c}$ is similar to the $s \bar{s}$ case, and therefore in the rest of the article we focus on the cases $q=b$ and $q=s$. The positivity of the coefficient $\rho_{1 q \bar{q}}$ indicates constructive interference between the contact interaction and resonant diagrams (for $\lambda_{H S}>0$ ). For an extended fit of the ratio $\rho$, including additional diagrams with the production of an intermediate Higgs boson due to $\mathcal{S}|H|^{2,4}$ interactions that turn out to be subdominant, see Appendix B.

The fact that for quark-antiquark annihilation the $h S$ process is nonvanishing for all values of the portal coupling $\lambda_{H S}$ indicates that one could employ this final state to exclude $b \bar{b}, s \bar{s}$, or $c \bar{c}$ annihilation as the dominant production process. The analysis that will follow in the present article suggests, however, that the diphoton decay of the $S$ alone may not be sufficient for that purpose for the benchmark points that we consider.

We show in Fig. 4 the variation of the ratio $\rho$ with the mass of the resonance, $M$, for the $g g$-initiated process and $\lambda_{H S}=1$, and for the $b \bar{b}$-initiated process for $\lambda_{H S}=0$ (no portal) and $\lambda_{H S}=1$. We have fixed the width to $\Gamma=1 \mathrm{GeV}$. Interestingly, the pure $q \bar{q}$-induced processes exhibit an increase of the ratio $\rho$ with increasing massrelated to the new $q \bar{q} h S$ interaction growing with momentum-whereas the pure $g g$-induced process exhibits a slight decrease.

If the diphoton resonance is wide, the analyses performed for the $h S$ final state will differ in the details due to changes in the kinematics. We show in Fig. 5 the variation of the ratio $\rho$ with the width over the mass, $\Gamma / M$, at a fixed

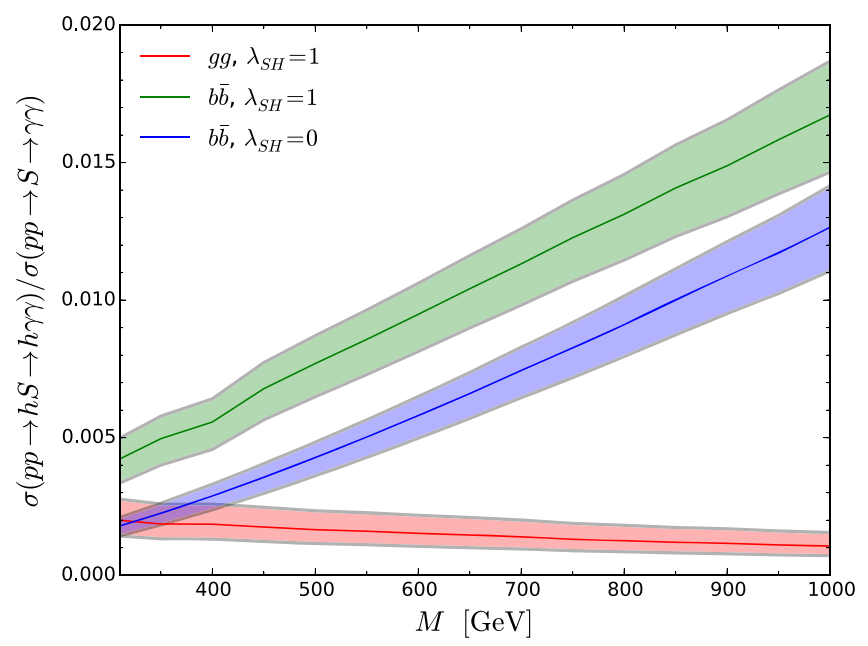

FIG. 4. The ratios $\rho(g g)$ and $\rho(b \bar{b})$ for $g g$ and $b \bar{b}$ initial states, defined between the associated production $p p \rightarrow h S \rightarrow h \gamma \gamma$ and the single production $p p \rightarrow S \rightarrow \gamma \gamma$, as functions of the mass of the resonance, for $\Gamma=1 \mathrm{GeV}$. The bands display the parton density function uncertainty for the MMHT14nlo68Cl set combined in quadrature with the scale variation between 0.5 and 2.0 times the default central dynamical scale implemented in MadGraph5_aMC@NLO.

mass $M=750 \mathrm{GeV}$, for $\lambda_{H S}=1$, and for the $b \bar{b}$-initiated process for $\lambda_{H S}=0$ (no portal) and $\lambda_{H S}=1$. One can observe that the central value of the ratio remains approximately constant in all cases, with only a slight decrease with increasing width.

In both Figs. 4 and 5, we also provide, as colored bands, the parton density function uncertainty for the MMHT14nlo68cl set [48] combined in quadrature with the scale variation between 0.5 and 2.0 times the default central dynamical scale implemented in MadGraph5_aMC@NLO. For a mass of $750 \mathrm{GeV}$, the total theoretical uncertainties due to scale and parton density function (PDF) variations are $\sim_{-30}^{+40} \%$ for the $g g$ induced process, $\sim \pm 10 \%$ for the $b \bar{b}$-induced case, and $\sim \pm 30 \%$ for the $s \bar{s}$-induced case (the latter is not shown in the figure for simplicity).

Assuming a total cross section for the production of a $\gamma \gamma$ resonance of mass $M=750 \mathrm{GeV}$ of, say, $\sigma(p p \rightarrow S \rightarrow$ $\gamma \gamma)=5 \mathrm{fb}$ (see below), one would expect a total of $\mathcal{O}(20)$ $h S \rightarrow h \gamma \gamma$ events at the high-luminosity LHC (HL-LHC, assuming $3000 \mathrm{fb}^{-1}$ of integrated luminosity) if the process is gluon-fusion initiated and $\mathcal{O}(200)$ events for $b \bar{b}$-initiated production, for a portal coupling $\lambda_{H S}=1$. Moreover, the minimum expected number of events for the $b \bar{b}$-initiated process is $\mathcal{O}(80)$, arising for $\lambda_{H S} \simeq-1.8$, and for the $s \bar{s}$ initiated process one expects a minimum of $\mathcal{O}(100)$ events for $\lambda_{H S} \simeq-2.0$. We note here that the positions of the minima for the $q \bar{q}$-initiated process will change after cuts due to the varying effect of the analysis on the different pieces contributing to the cross section. 


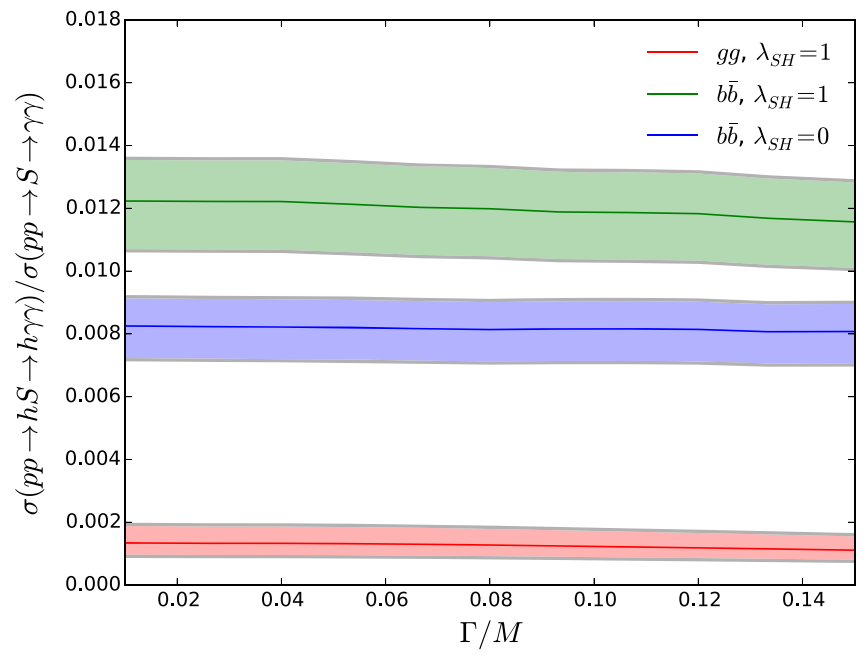

FIG. 5. The ratios $\rho(g g)$ and $\rho(b \bar{b})$ for $g g$ and $b \bar{b}$ initial states, defined between the associated production $p p \rightarrow h S \rightarrow h \gamma \gamma$ and the single production $p p \rightarrow S \rightarrow \gamma \gamma$, as functions of the width of the resonance over the mass, $\Gamma / M$. The bands display the parton density function uncertainty for the MMHT14nlo68cl set combined in quadrature with the scale variation between 0.5 and 2.0 times the default central dynamical scale implemented in MadGraph5 aMC@NLO. The scalar resonance mass was chosen to be $M=7 \overline{50} \mathrm{GeV}$.

The kinematic structure of the $p p \rightarrow h S \rightarrow h \gamma \gamma$ process can be well described by examining the distribution of the invariant mass of the $\gamma \gamma$ state, $M_{\gamma \gamma}$, or the distribution of the invariant mass of the Higgs boson and diphoton combination, $M_{h \gamma \gamma}$. In Figs. 6 and 7 we show, respectively, these distributions for the gluon-fusion-initiated process, for two widths, $\Gamma=1 \mathrm{GeV}$ and $\Gamma=45 \mathrm{GeV}$. For the sake of clarity, here we only show distributions for a scalar mass

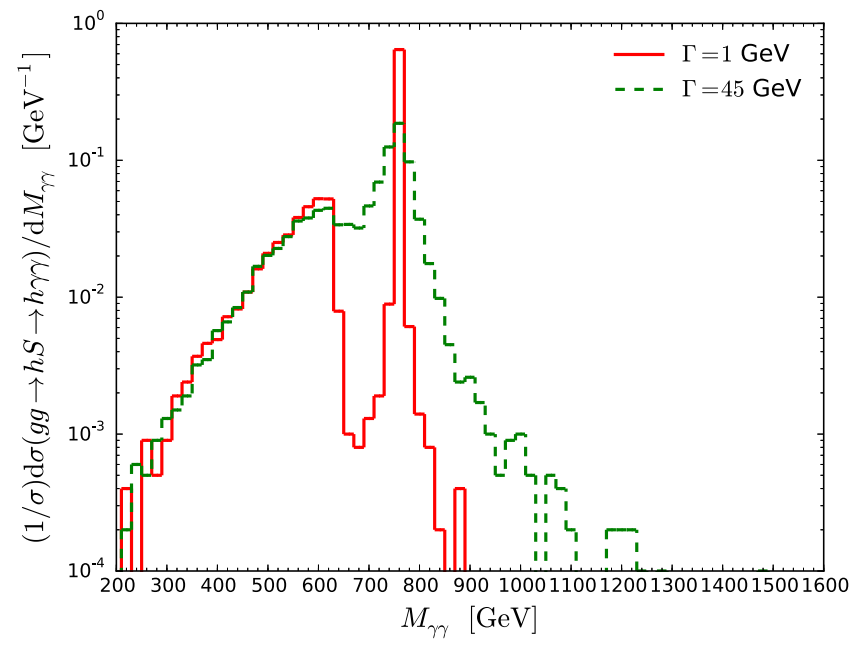

FIG. 6. The matrix-element level distribution of the diphoton invariant mass, $M_{\gamma \gamma}$, in the $g g \rightarrow h S \rightarrow h \gamma \gamma$ process, normalized to unity, for the two different width scenarios, $\Gamma=1 \mathrm{GeV}$ and $\Gamma=45 \mathrm{GeV}$, for $M=750 \mathrm{GeV}$.

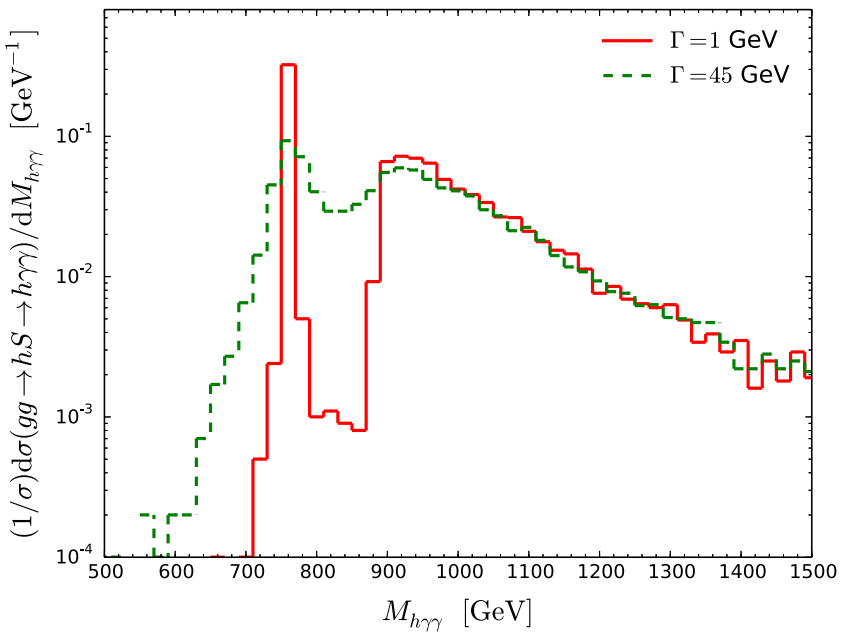

FIG. 7. The matrix-element-level distribution of the combined Higgs boson and diphoton invariant mass, $M_{h \gamma \gamma}$, in the $g g \rightarrow h S \rightarrow h \gamma \gamma$ process, normalized to unity, for the two different width scenarios, $\Gamma=1 \mathrm{GeV}$ and $\Gamma=45 \mathrm{GeV}$, for $M=750 \mathrm{GeV}$.

of $M=750 \mathrm{GeV}$, but the main features remain unaltered as long as the scalar is heavier than the Higgs boson, $M>M_{h}$. The distributions clearly show the existence of two regions: a region in which the intermediate $s$-channel propagator for the $S$ scalar is on shell and the final-state $S(\gamma \gamma)$ is off shell, and a region in which the $s$-channel internal propagator is instead off shell and the final-state $S(\gamma \gamma)$ is on shell. The existence of the former region, $M_{\gamma \gamma} \lesssim M-M_{h}, M_{h \gamma \gamma} \sim M$ which henceforth we will call "three-body decay" since the intermediate $S$ is decaying approximately on shell, is made possible by the fact that the mass of the particle produced in association with

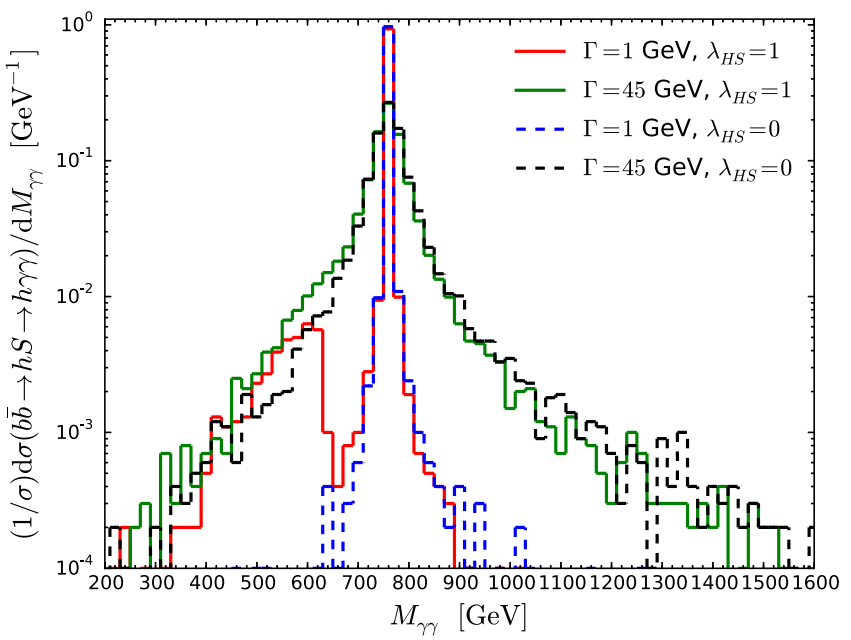

FIG. 8. The matrix-element-level distribution of the diphoton invariant mass, $M_{\gamma \gamma}$, in the $b \bar{b} \rightarrow h S \rightarrow h \gamma \gamma$ process, normalized to unity, for two width scenarios, $\Gamma=1,45 \mathrm{GeV}$, and two values of the portal coupling, $\lambda_{H S}=0,1$, for $M=750 \mathrm{GeV}$. 


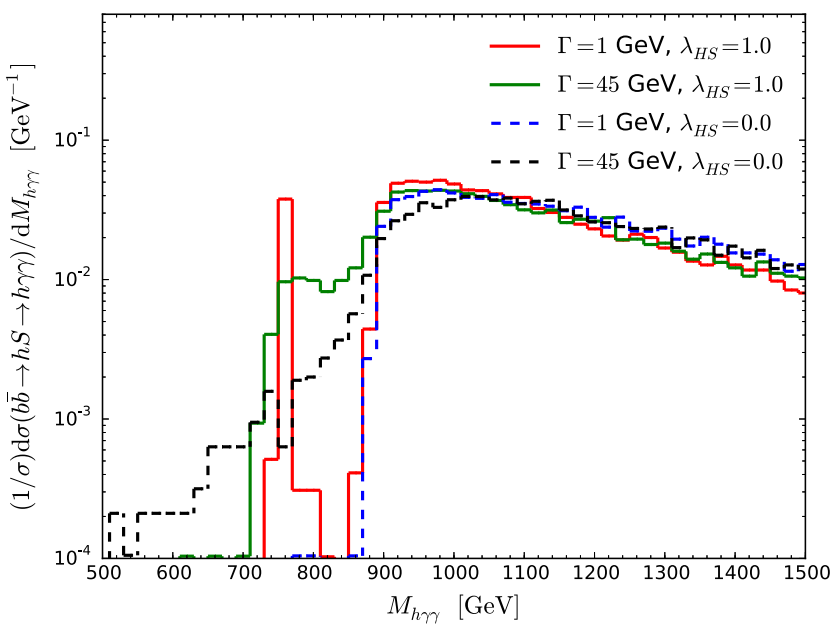

FIG. 9. The matrix-element level distribution of the combined Higgs boson and diphoton invariant mass, $M_{h \gamma \gamma}$, in the $b \bar{b} \rightarrow$ $h S \rightarrow h \gamma \gamma$ process, normalized to unity, for two width scenarios, $\Gamma=1,45 \mathrm{GeV}$, and two values of the portal coupling, $\lambda_{H S}=0,1$, for $M=750 \mathrm{GeV}$.

$S$, the Higgs boson, is smaller than the masses of $S$ we are considering, $M>M_{h}=125 \mathrm{GeV}$. The other region, $M_{\gamma \gamma} \sim M, M_{h \gamma \gamma} \gtrsim M+M_{h}$ which we will refer to as "onshell diphoton," exists irrespective of the mass of $S$. Note that both the three-body decay and on-shell diphoton regions exist even for $\Gamma / M \ll 1$. The normalized distributions look identical for all values of the portal coupling, $\lambda_{H S}$ $(\neq 0)$, since the dominant contribution stems by far from the diagram shown in Fig. 1(a).

In Figs. 8 and 9 we show the diphoton invariant mass and the combined Higgs boson and diphoton invariant mass for the $b \bar{b}$-initiated process, respectively, for $M=750 \mathrm{GeV}$. Evidently the two regions observed for the $g g$ case are

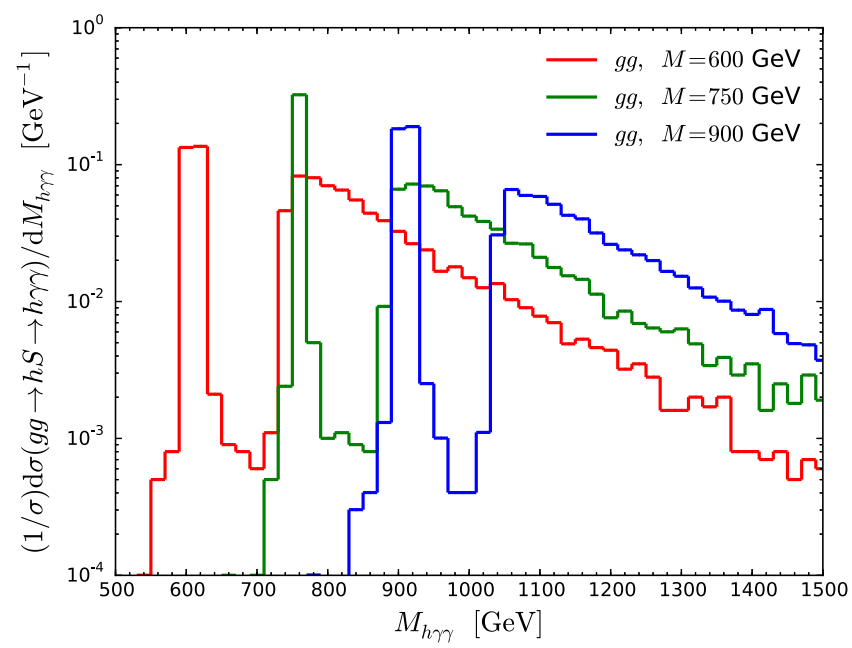

FIG. 10. The matrix-element level distribution of the combined Higgs boson and diphoton invariant mass, $M_{h \gamma \gamma}$, in the $g g \rightarrow$ $h S \rightarrow h \gamma \gamma$ process, normalized to unity, for three benchmark mass scenarios, $M=600,750,900 \mathrm{GeV}$, and $\Gamma=1 \mathrm{GeV}$.

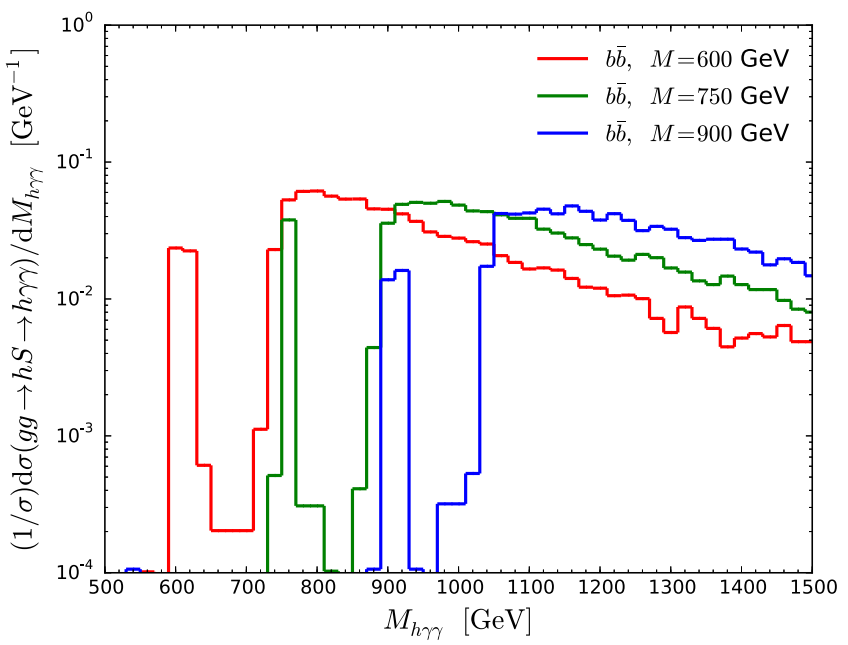

FIG. 11. The matrix-element level distribution of the combined Higgs boson and diphoton invariant mass, $M_{h \gamma \gamma}$, in the $b \bar{b} \rightarrow$ $h S \rightarrow h \gamma \gamma$ process, normalized to unity, for three benchmark mass scenarios, $M=600,750,900 \mathrm{GeV}$, and $\Gamma=1 \mathrm{GeV}$, for $\lambda_{H S}=1$.

clearly still present for $\lambda_{H S} \neq 0$ and $\Gamma=1 \mathrm{GeV}$, with the on-shell diphoton region dominating. For $\lambda_{H S}=0$, the "three-body decay" region disappears completely since the resonant $s$-channel diagram of Fig. 2(a) vanishes. For large width the two regions merge into one, and the effect of the vanishing three-body decay region for $\lambda_{H S}=0$ is not as evident as in the case of small width. The distributions for the $s \bar{s}$-initiated process exhibit similar features, with different "mixtures" between the two regions arising from the differences between the strange and bottom quark parton density functions. We omit them for the sake of simplicity.

Figures 10 and 11 show the distributions of the combined invariant mass of the Higgs boson and diphoton, $M_{h \gamma \gamma}$, for the pure $g g$-initiated and pure $b \bar{b}$-initiated cases, respectively, for the three values of the scalar mass that we will consider as "benchmark" scenarios in our analysis, $M=600,750,900 \mathrm{GeV}$ (see below) and $\Gamma=1 \mathrm{GeV}$. For the $b \bar{b}$ case we only show the $\lambda_{H S}=1$ distributions for simplicity. They all clearly demonstrate the existence of the main features described for the $M=750 \mathrm{GeV}$ case, i.e., the three-body decay and on-shell diphoton regions.

\section{EVENT GENERATION AND DETECTOR SIMULATION}

\section{A. Event generation}

The signal model was generated via an implementation of the Lagrangian of Eq. (1) in FeynRules [49,50]. Via the Universal FeynRules Output (UFO) interface [51] this was used to generate parton-level events employing MadGraph5_aMC@NLO [52,53]. The background processes were also generated using MadGraph5_aMC@NLO, with appropriate generation-level cuts to reduce the initial 
cross sections to a manageable level. All the events were passed through the HERWIG 7 [54-58] Monte Carlo generator for simulation of the parton shower, the underlying event, and hadronization. As before, the MMHT14nlo68cl PDF set was employed. To remain conservative, we consider collisions at the LHC at a centerof-mass energy of $13 \mathrm{TeV}$. The possible increase of energy to $14 \mathrm{TeV}$ will increase rates in the considered processes by $\mathcal{O}(10 \%)$.

Since we expect to impose cuts on the diphoton mass window, $M_{\gamma \gamma}$, that are sufficiently far away from the Higgs boson resonance, we can immediately exclude any background processes containing $h \rightarrow \gamma \gamma$ from the analysis. For this reason we do not include associated Higgs boson production with a vector boson or Higgs boson pair production, $t \bar{t} h$ production, and so on. This implies that the relevant backgrounds are those with nonresonant $\gamma \gamma$ production, other processes that involve $S \rightarrow \gamma \gamma$, and reducible backgrounds. We thus consider the following processes: $\gamma+$ jets, $\gamma \gamma+$ jets, events with at least one true $b$ quark at parton level $(b+$ jets), $Z \gamma \gamma$ with $Z \rightarrow b \bar{b}, t \bar{t} \gamma \gamma$ including all the decay modes of the top quarks, and the production of the resonance $S$ in association with a nonresonant $b \bar{b}$ pair. ${ }^{10}$ All the multijet processes are generated without merging to the parton shower, in the five-flavor scheme, with four outgoing partons at the matrix-element level.

The calculation of higher-order QCD corrections to these multileg processes, particularly when restricting the phase space with cuts, is numerically challenging at present. To remain conservative, we will assume that the corrections are large and apply $K$ factors of $K=2$ to all the background processes. For the signal and the $b \bar{b} S$ associated production we do not apply any $K$ factors since the corrections are approximately absorbed into the ratio with the single inclusive production of the $S$ resonance; see below. Throughout this article we assume that $\sigma(p p \rightarrow$ $S \rightarrow \gamma \gamma)=10,5,1 \mathrm{fb}$, corresponding to the benchmark masses $M=600,750,900 \mathrm{GeV}$, fixing the product $c_{G}^{S} c_{\gamma}^{S}$ [or $\left(y_{d}^{S}\right)^{i i} c_{\gamma}^{S}$ ] which drops out in the ratio $\rho$. The values of the cross sections are motivated by the current ATLAS [16] and CMS [17] limits on diphoton resonances.

Note that it turns out that the nonresonant $b \bar{b} S$ process is only relevant for gluon-fusion production of $S$, and we only report numbers for that in what follows.

\section{B. Detector simulation}

In the hadron-level analysis that follows, performed without using any dedicated detector simulation software, we consider all particles within a pseudorapidity of $|\eta|<5$

\footnotetext{
${ }^{10} \mathrm{We}$ also considered the $h \gamma \gamma$ process, including the loopinduced pieces [59], but found that it possesses a negligible cross section.
}

and $p_{T}>100 \mathrm{MeV}$. We smear the momenta of all reconstructed objects (i.e., jets, electrons, muons, and photons) according to HL-LHC projections [60,61]. We also apply the relevant reconstruction efficiencies. We simulate $b$-jet tagging by looking for jets containing $B$ hadrons, which we have set to stable in the simulation, and considering them as the $b$-jet candidates. The mistagging of $c$ jets to $b$ jets is performed by choosing $c$-jet candidates (after hadronization) as those jets that lie within a distance $\Delta R<0.4$ from $c$ quarks (after the parton shower), with transverse momentum $p_{T}>1 \mathrm{GeV} .{ }^{11}$ We apply a flat $b$-tagging efficiency of $70 \%$ and a mistag rate of $1 \%$ for light-flavor jets and $10 \%$ for charm-quark-initiated jets.

We reconstruct jets using the anti- $k_{t}$ algorithm available in the FastJet package $[62,63]$, with a radius parameter of $R=0.4$. We only consider jets, photons, and leptons with $p_{T}>30 \mathrm{GeV}$ within pseudorapidity $|\eta|<2.5$ in our analysis. The jet-to-lepton misidentification probability is taken to be $\mathcal{P}_{j \rightarrow \ell}=0.0048 \times \mathrm{e}^{-0.035 p_{T j} / \mathrm{GeV}}$, and the jetto-photon misidentification probability was taken to be $\mathcal{P}_{j \rightarrow \gamma}=0.0093 \times \mathrm{e}^{-0.036 p_{T j} / \mathrm{GeV}}[60,61]$, both flat in pseudorapidity. We demand all leptons and photons to be isolated, where an isolated object is defined to have $\sum_{i} p_{T, i}$ less than $15 \%$ of its transverse momentum in a cone of $\Delta R=0.2$ around it.

\section{DETAILED ANALYSIS}

We consider events with two reconstructed $b$ jets and two isolated photons as defined in Sec. III. Note that this final state has been previously considered in the context of searches for Higgs boson pair production, e.g. in [64-68]. We impose the following "acceptance" cuts to all samples:

(i) $b$ jets: transverse momenta $p_{T, b 1}>30 \mathrm{GeV}$, $p_{T, b 2}>30 \mathrm{GeV}$, all $b$ jets within $|\eta|<2.5$,

(ii) photons: transverse momenta $p_{T, \gamma 1}>30 \mathrm{GeV}$, $p_{T, \gamma 2}>30 \mathrm{GeV}$, all photons within $|\eta|<2.5$,

(iii) invariant mass of the two $b$-jets $M_{b \bar{b}} \in$ $[90,160] \mathrm{GeV}$,

(iv) invariant mass of the two photons $M_{\gamma \gamma}>M-$ $300 \mathrm{GeV}$,

(v) veto events with leptons of $p_{T}>25 \mathrm{GeV}$ within $|\eta|<2.5$,

for each of the considered diphoton resonance masses, $M$.

The cross sections after application of the acceptance cuts are given in Table I for two values of the widths $\Gamma=$ $1 \mathrm{GeV}$ and $\Gamma=45 \mathrm{GeV}$ and for $M=750 \mathrm{GeV}$. For the case of $q \bar{q}$ we consider as examples $\lambda_{H S}=1$ and $\lambda_{H S}=0$. Throughout this analysis, the total signal cross section was calculated by using the ratio $\rho$ (derived in Sec. II) as $\sigma(p p \rightarrow h S \rightarrow h \gamma \gamma)=\rho \times \sigma(p p \rightarrow S \rightarrow \gamma \gamma)$, where $\sigma(p p \rightarrow S \rightarrow \gamma \gamma)=10,5, \quad 1 \mathrm{fb}$ for $M=600,750$,

\footnotetext{
${ }^{11}$ This procedure of associating jets to $c$ quarks is expected to be conservative.
} 
TABLE I. The expected cross sections at $13 \mathrm{TeV} p p$ collision energy for all the considered processes after acceptance cuts for $M=750 \mathrm{GeV}$ and $\Gamma=1,45 \mathrm{GeV}$. All branching ratios, acceptances, and tagging rates have been applied. We have assumed that the single production cross section for a diphoton scalar resonance of $M=750 \mathrm{GeV}$ is $\sigma(p p \rightarrow S \rightarrow \gamma \gamma)=5 \mathrm{fb}$.

\begin{tabular}{lr} 
Process & Acceptance $\sigma$ \\
\hline$g g \rightarrow h(b \bar{b}) S(\gamma \gamma), \Gamma=1 \mathrm{GeV}, \lambda_{H S}=1$ & 0.00054 \\
$g g \rightarrow h(b \bar{b}) S(\gamma \gamma), \Gamma=45 \mathrm{GeV}, \lambda_{H S}=1$ & 0.00055 \\
$b \bar{b} \rightarrow h(b \bar{b}) S(\gamma \gamma), \Gamma=1 \mathrm{GeV}, \lambda_{H S}=1$ & 0.00266 \\
$b \bar{b} \rightarrow h(b \bar{b}) S(\gamma \gamma), \Gamma=45 \mathrm{GeV}, \lambda_{H S}=1$ & 0.00254 \\
$b \bar{b} \rightarrow h(b \bar{b}) S(\gamma \gamma), \Gamma=1 \mathrm{GeV}, \lambda_{H S}=0$ & 0.00184 \\
$b \bar{b} \rightarrow h(b \bar{b}) S(\gamma \gamma), \Gamma=45 \mathrm{GeV}, \lambda_{H S}=0$ & 0.00172 \\
$s \bar{s} \rightarrow h(b \bar{b}) S(\gamma \gamma), \Gamma=1 \mathrm{GeV}, \lambda_{H S}=1$ & 0.00366 \\
$s \bar{s} \rightarrow h(b \bar{b}) S(\gamma \gamma), \Gamma=45 \mathrm{GeV}, \lambda_{H S}=1$ & 0.00370 \\
$s \bar{s} \rightarrow h(b \bar{b}) S(\gamma \gamma), \Gamma=1 \mathrm{GeV}, \lambda_{H S}=0$ & 0.00291 \\
$s \bar{s} \rightarrow h(b \bar{b}) S(\gamma \gamma), \Gamma=45 \mathrm{GeV}, \lambda_{H S}=0$ & 0.00249 \\
$\mathrm{At}$ least one $b$ quark $+\mathrm{jets}$ & 0.31300 \\
$\gamma+\mathrm{jets}$ & 0.11259 \\
$\gamma \gamma+$ jets & 0.15766 \\
$Z \gamma \gamma \rightarrow(b \bar{b}) \gamma \gamma$ & 0.00489 \\
$t \bar{t} \gamma \gamma$ & 0.00281 \\
$g g \rightarrow b \bar{b} S(\gamma \gamma), \Gamma=1 \mathrm{GeV}$ & 0.00058 \\
$g g \rightarrow b \bar{b} S(\gamma \gamma), \Gamma=45 \mathrm{GeV}$ & 0.00063
\end{tabular}

$900 \mathrm{GeV}$, and including the decay $h \rightarrow b \bar{b}$. This cross section was employed as the normalization of the signal event samples (before analysis and acceptance cuts). The expected number of signal events, for $\lambda_{H S} \sim \mathcal{O}(1)$, after acceptance cuts is $\mathcal{O}(1)-\mathcal{O}(10)$ at $3000 \mathrm{fb}^{-1}$ of integrated luminosity. However, as already discussed, one should keep in mind that the cross section grows with $\lambda_{H S}^{2}$ in both $g g$ - and $q \bar{q}$-initiated production.

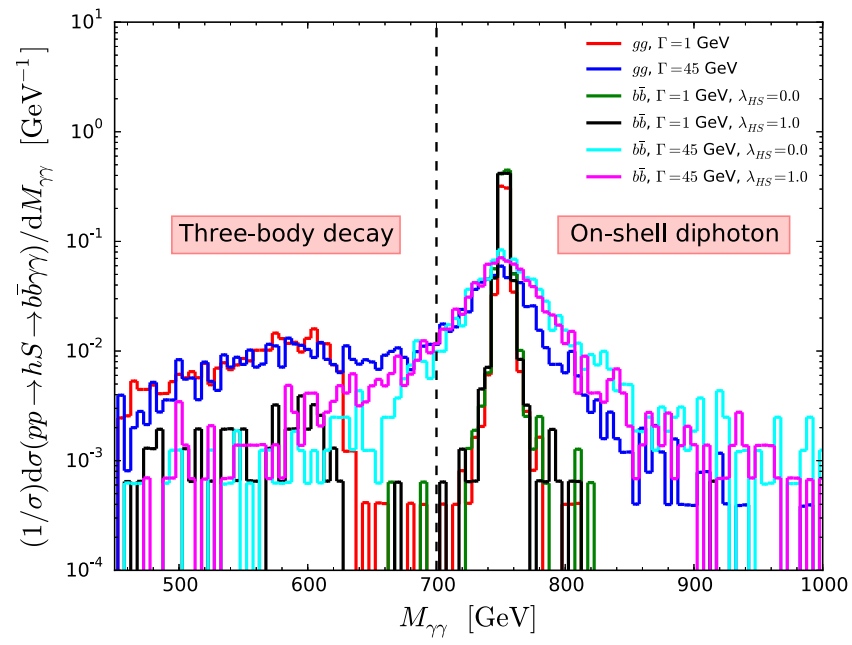

FIG. 12. The distribution of the diphoton invariant mass, $M_{\gamma \gamma}$, for the $g g \rightarrow h S \rightarrow b \bar{b} \gamma \gamma$ process after acceptance cuts, normalized to unity, for two width scenarios, $\Gamma=1 \mathrm{GeV}$ and $\Gamma=45 \mathrm{GeV}$, while $M=750 \mathrm{GeV}$.

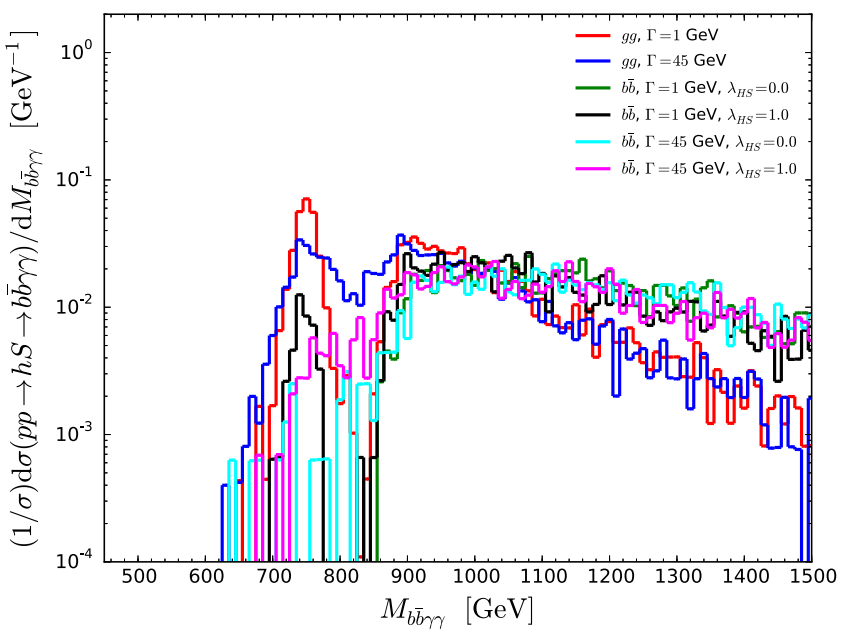

FIG. 13. The distribution of the combined diphoton $+b \bar{b}$ invariant mass, $M_{b \bar{b} \gamma \gamma}$, for the $g g \rightarrow h S \rightarrow b \bar{b} \gamma \gamma$ process after acceptance cuts, normalized to unity, for two width scenarios, $\Gamma=1 \mathrm{GeV}$ and $\Gamma=45 \mathrm{GeV}$, while $M=750 \mathrm{GeV}$.

The resulting diphoton invariant mass after acceptance cuts is shown in Fig. 12 for the example of $M=750 \mathrm{GeV}$. The $M_{\gamma \gamma}$ observable can be used to separate the analysis into the two regions described in Sec. II: the three-body decay region (TBD) and the on-shell diphoton region $(\mathrm{OS} \gamma \gamma)$. The separation is identical in both $g g$ - and $q \bar{q}$ initiated processes. We choose $M_{\gamma \gamma}<M-50 \mathrm{GeV}$ for the TBD region and $M_{\gamma \gamma}>M-50 \mathrm{GeV}$ for the $\mathrm{OS} \gamma \gamma$ region for a diphoton resonance mass, $M$. We also show the distribution of the combined invariant mass of the two $b$-jet candidates and the diphoton system, $M_{b \bar{b} \gamma \gamma}$ in Fig. 13, which also clearly demonstrates the existence of the two regions.

We sapply further cuts to improve signal and background discrimination. As we did not attempt to fully optimize the cuts in the present analysis, we apply a common set of cuts along with invariant mass cuts on the observables $M_{\gamma \gamma}$ and $M_{b \bar{b} y \gamma}$ that provide the main distinction between the two regions. The common cuts applied in each region are shown

TABLE II. The additional common cuts applied along with the acceptance cuts in both the three-body decay and the on-shell diphoton region for the $g g$ - and $q \bar{q}$-initiated processes. The labels " 1 " and " 2 " correspond to the hardest and second hardest reconstructed objects (photons or $b$ jets), respectively.

\begin{tabular}{lc}
\hline \hline Observable & Cut \\
\hline$p_{T, \gamma 1}$ & $>200 \mathrm{GeV}$ \\
$p_{T, \gamma 2}$ & $>120 \mathrm{GeV}$ \\
$\Delta R(\gamma, \gamma)$ & $\in[2.0,4.0]$ \\
$M_{b \bar{b}}$ & $\in[100,150] \mathrm{GeV}$ \\
$\Delta R(b, \bar{b})$ & $\in[0.8,3.0]$ \\
$\Delta R\left(\gamma \gamma, b_{2}\right)$ & $<3.0$ \\
\hline \hline
\end{tabular}


TABLE III. The additional invariant mass cuts applied along with the further cuts of Table II, in the three-body decay region and the on-shell diphoton region, for both the $g g$ - and $q \bar{q}$-initiated processes for a scalar diphoton resonance of mass $M$. The different choices for the mass windows were made according to the width of the resonance, $\Gamma$.

\begin{tabular}{lcc}
\hline \hline & \multicolumn{1}{c}{ TBD } & OS $\gamma \gamma$ \\
\hline$\Gamma=1 \mathrm{GeV}$ & & \\
$M_{\gamma \gamma}$ & $\in M_{-300}^{-110} \mathrm{GeV}$ & $\in M \pm 5 \mathrm{GeV}$ \\
$M_{b \bar{b} \gamma \gamma}$ & $\in M \pm 30 \mathrm{GeV}$ & $\cdots$ \\
$\Gamma=45 \mathrm{GeV}$ & & \\
$M_{\gamma \gamma}$ & $\in M_{-300}^{-110} \mathrm{GeV}$ & $\in M \pm 40 \mathrm{GeV}$ \\
$M_{b \bar{b} \gamma \gamma}$ & $\in M \pm 40 \mathrm{GeV}$ & $\cdots$ \\
\hline \hline
\end{tabular}

in Table II, and the specific invariant mass cuts are shown in Table III. Effectively, the cuts aim to exploit the fact that the photons in the signal are harder than in the backgrounds and also feature tighter diphoton and $b \bar{b}$ mass windows, particularly in the OS $\gamma \gamma$ region for the former.

We show the resulting cross sections after the application of these further cuts in Table IV, for the case of $M=750 \mathrm{GeV}$. A high efficiency is maintained for the signal, with high rejection factors for the background processes. We note again that the $b \bar{b} S$ associated production process is relevant only for the gluon-fusion scenario.

To obtain the $95 \%$ confidence-level exclusion regions for $\lambda_{H S}$ we use Poissonian statistics to calculate the

TABLE IV. The expected cross sections at $13 \mathrm{TeV} p p$ collision energy in fb for all the considered processes after the application of further cuts as in Tables II and III, for $M=750 \mathrm{GeV}$ and $\Gamma=1,45 \mathrm{GeV}$. All branching ratios, acceptances, and tagging rates have been applied. We have assumed that the single production cross section for a diphoton scalar resonance of $M=750 \mathrm{GeV}$ is $\sigma(p p \rightarrow S \rightarrow \gamma \gamma)=5 \mathrm{fb}$.

\begin{tabular}{lcc}
\hline \hline Process & TBD & OS $\gamma \gamma$ \\
\hline$g g \rightarrow h(b \bar{b}) S(\gamma \gamma), \Gamma=1 \mathrm{GeV}, \lambda_{H S}=1$ & 0.00007 & 0.00020 \\
$g g \rightarrow h(b \bar{b}) S(\gamma \gamma), \Gamma=45 \mathrm{GeV}, \lambda_{H S}=1$ & 0.00006 & 0.00014 \\
$b \bar{b} \rightarrow h(b \bar{b}) S(\gamma \gamma), \Gamma=1 \mathrm{GeV}, \lambda_{H S}=1$ & 0.00007 & 0.00105 \\
$b \bar{b} \rightarrow h(b \bar{b}) S(\gamma \gamma), \Gamma=45 \mathrm{GeV}, \lambda_{H S}=1$ & 0.00038 & 0.00074 \\
$b \bar{b} \rightarrow h(b \bar{b}) S(\gamma \gamma), \Gamma=1 \mathrm{GeV}, \lambda_{H S}=0$ & 0 & 0.00064 \\
$b \bar{b} \rightarrow h(b \bar{b}) S(\gamma \gamma), \Gamma=45 \mathrm{GeV}, \lambda_{H S}=0$ & 0.00018 & 0.00055 \\
$s \bar{s} \rightarrow h(b \bar{b}) S(\gamma \gamma), \Gamma=1 \mathrm{GeV}, \lambda_{H S}=1$ & 0.00005 & 0.00134 \\
$s \bar{s} \rightarrow h(b \bar{b}) S(\gamma \gamma), \Gamma=45 \mathrm{GeV}, \lambda_{H S}=1$ & 0.00005 & 0.00107 \\
$s \bar{s} \rightarrow h(b \bar{b}) S(\gamma \gamma), \Gamma=1 \mathrm{GeV}, \lambda_{H S}=0$ & 0 & 0.00101 \\
$s \bar{s} \rightarrow h(b \bar{b}) S(\gamma \gamma), \Gamma=45 \mathrm{GeV}, \lambda_{H S}=0$ & 0.00003 & 0.00072 \\
At least one $b$ quark $+\mathrm{jets}$ & $\mathcal{O}\left(10^{-7}\right)$ & $\mathcal{O}\left(10^{-10}\right)$ \\
$\gamma+$ jets & 0.00025 & $\mathcal{O}\left(10^{-7}\right)$ \\
$\gamma \gamma+$ jets & 0.00182 & 0.00003 \\
$Z \gamma \gamma \rightarrow(b \bar{b}) \gamma \gamma$ & $6 \times 10^{-5}$ & $10^{-5}$ \\
$t \bar{t} \gamma \gamma$ & $9 \times 10^{-5}$ & $10^{-5}$ \\
$g g \rightarrow b \bar{b} S(\gamma \gamma), \Gamma=1 \mathrm{GeV}$ & $\lesssim \mathcal{O}\left(10^{-6}\right)$ & 0.00020 \\
$g g \rightarrow b \bar{b} S(\gamma \gamma), \Gamma=45 \mathrm{GeV}$ & $\mathcal{O}\left(10^{-6}\right)$ & 0.00018 \\
\hline \hline
\end{tabular}

probabilities. Since we have assumed that the production of a scalar diphoton resonance will have been observed, we have to construct a null hypothesis compatible with such an observation providing the expected number of events at the LHC that we will confront with the theory predictions in the parameter space to be tested. If these numbers differ by a certain significance, the corresponding point is expected to be excluded with this significance. In particular, any hypothesis has to be realistic and remain within the bounds of our model. Our underlying assumption is thus chosen to be that the scalar resonance $S$ is produced purely in gluon fusion to good approximation and that there is no portal coupling, $\lambda_{H S}=0$, which means there is basically no $h+S$ associated production. For further technical details on this statistical procedure, see Appendix C of Ref. [69]. We do not incorporate the effect of systematic uncertainties on the signal or backgrounds. To perform a combination of the two analysis regions, TBD and $O S \gamma \gamma$, we employ the "Stouffer method" [70], where the combined significance, $\Omega$, is given, in terms of the individual significances $\Omega_{i}$, as

$$
\Omega=\frac{1}{\sqrt{k}} \sum_{i=1}^{k} \Omega_{i}
$$

We show the resulting expected limits (assuming our null hypothesis is true) as a function of the integrated luminosity for the different benchmark scenarios that we consider in Figs. 14-25. For the case $M=750 \mathrm{GeV}$ we show results for $\Gamma=45 \mathrm{GeV}$ as well. For $\Gamma=1 \mathrm{GeV}$, we obtain more stringent constraints, limiting, for $M=600,750,900 \mathrm{GeV}$, respectively, $\left|\lambda_{H S}\right| \lesssim 2,4,5$ for the $g g$-initiated process and

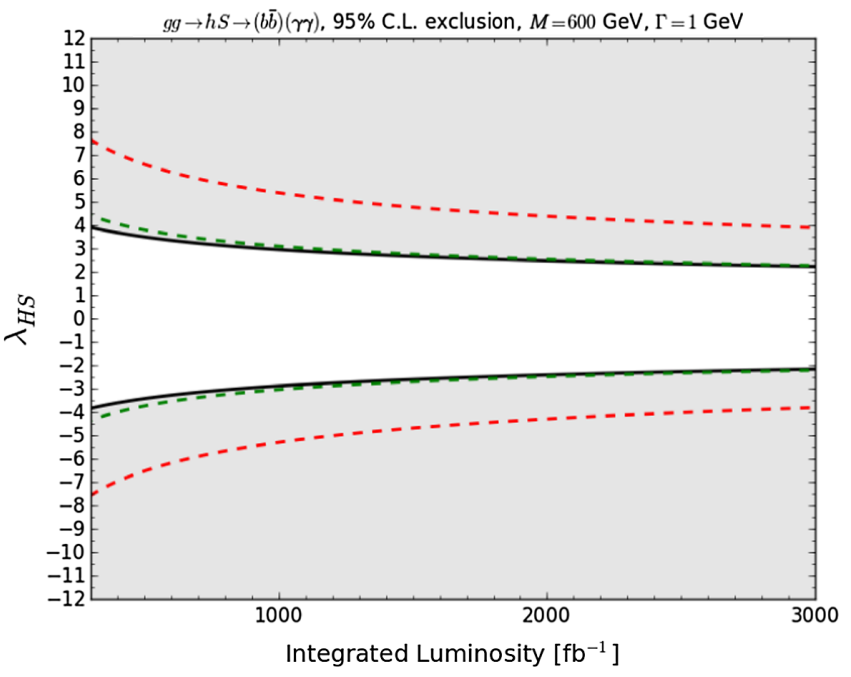

FIG. 14. The grey-shaded area shows the $95 \%$ confidence-level exclusion region for the portal coupling $\lambda_{H S}$ for the $g g$-induced case and $M=600 \mathrm{GeV}, \Gamma=1 \mathrm{GeV}$, for the combination of the TBD (red) and OS $\gamma \gamma$ (green) regions as defined in the main text. We have assumed that the single production cross section $\sigma(p p \rightarrow S \rightarrow \gamma \gamma)=10 \mathrm{fb}$. 


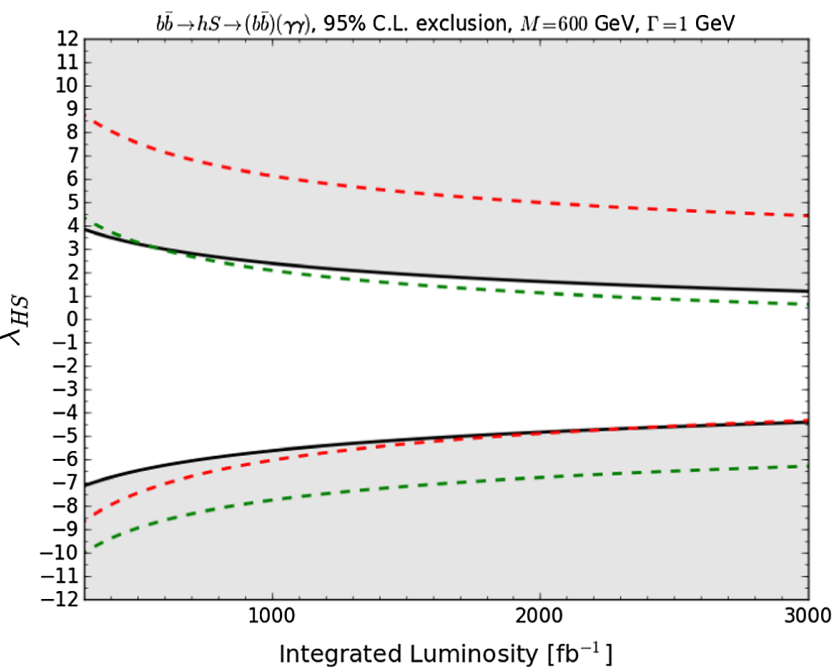

FIG. 15. The grey-shaded area shows the $95 \%$ confidence-level exclusion region for the portal coupling $\lambda_{H S}$ for the $b \bar{b}$-induced case and $M=600 \mathrm{GeV}, \Gamma=1 \mathrm{GeV}$, for the combination of the TBD (red) and OS $\gamma \gamma$ (green) regions as defined in the main text. We have assumed that the single production cross section $\sigma(p p \rightarrow S \rightarrow \gamma \gamma)=10 \mathrm{fb}$.

$\lambda_{H S} \in[\sim-4, \sim 1],[\sim-7, \sim 3],[\sim-8, \sim 4]$, for both the $b \bar{b}$ - and $s \bar{s}$-initiated processes, at the end of the HLLHC run $\left(3000 \mathrm{fb}^{-1}\right)$. The variation between the results for $b \bar{b}$ - and $s \bar{s}$-initiated processes-visible in the plots-is very small and can be attributed to the differences between the parton density functions for the strange and bottom quarks, as already mentioned.

The scenario with the larger width, $M=750 \mathrm{GeV}$, $\Gamma=45 \mathrm{GeV}$, clearly exhibits weaker constraints, with the

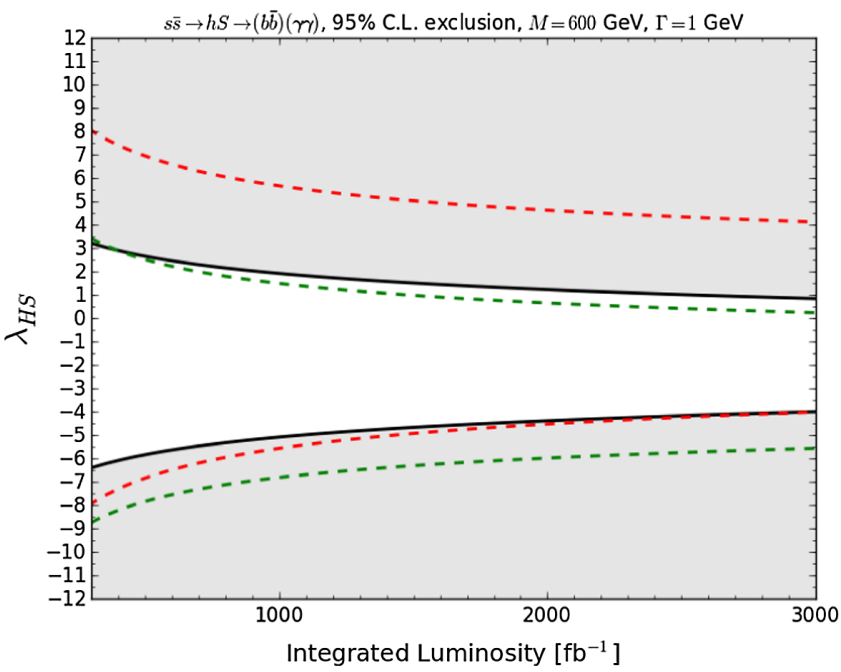

FIG. 16. The grey-shaded area shows the $95 \%$ confidence-level exclusion region for the portal coupling $\lambda_{H S}$ for the $s \bar{s}$-induced case and $M=600 \mathrm{GeV}, \Gamma=1 \mathrm{GeV}$, for the combination of the $\mathrm{TBD}$ (red) and OS $\gamma \gamma$ (green) regions as defined in the main text. We have assumed that the single production cross section $\sigma(p p \rightarrow S \rightarrow \gamma \gamma)=10 \mathrm{fb}$.

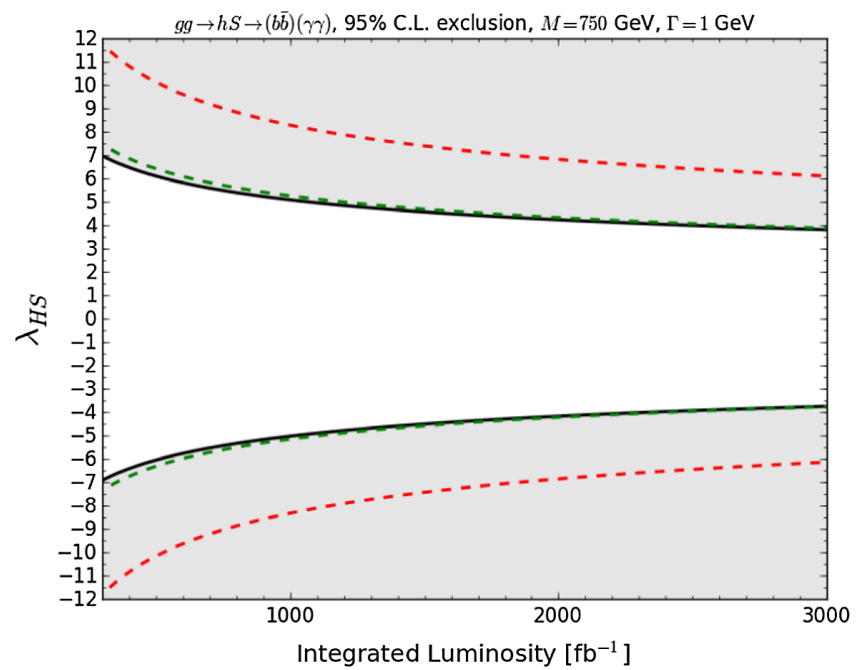

FIG. 17. The grey-shaded area shows the $95 \%$ confidence-level exclusion region for the portal coupling $\lambda_{H S}$ for the $g g$-induced case and $M=750 \mathrm{GeV}, \Gamma=1 \mathrm{GeV}$, for the combination of the TBD (red) and OS $\gamma \gamma$ (green) regions as defined in the main text. We have assumed that the single production cross section $\sigma(p p \rightarrow S \rightarrow \gamma \gamma)=5 \mathrm{fb}$.

$g g$-initiated processes yielding $\left|\lambda_{H S}\right| \lesssim 5$, the $b \bar{b}$-initiated process $\lambda_{H S} \in[\sim-8, \sim 5]$, and the $s \bar{s}$-initiated process $\lambda_{H S} \in[\sim-8, \sim 4]$. It is conceivable that if further decay channels of the resonance $S$ are discovered, the remaining unconstrained regions in the $q \bar{q}$ cases can be covered (in particular for narrow width), allowing determination of the initial state partons that produce the resonance.

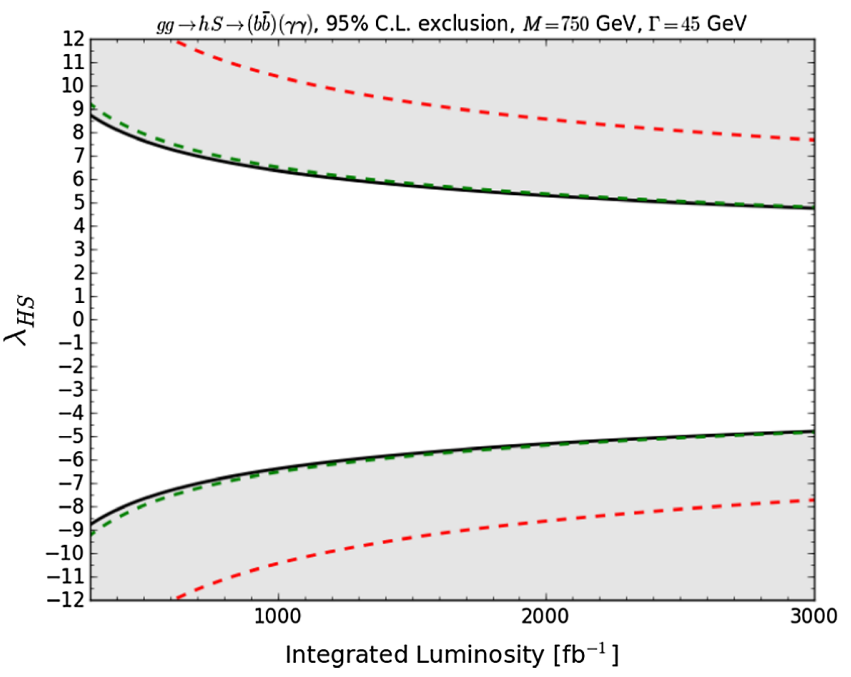

FIG. 18. The grey-shaded area shows the 95\% confidence-level exclusion region for the portal coupling $\lambda_{H S}$ for the $g g$-induced case and $M=750 \mathrm{GeV}, \Gamma=45 \mathrm{GeV}$, for the combination of the $\mathrm{TBD}$ (red) and $\mathrm{OS} \gamma \gamma$ (green) regions as defined in the main text. We have assumed that the single production cross section $\sigma(p p \rightarrow S \rightarrow \gamma \gamma)=5 \mathrm{fb}$. 


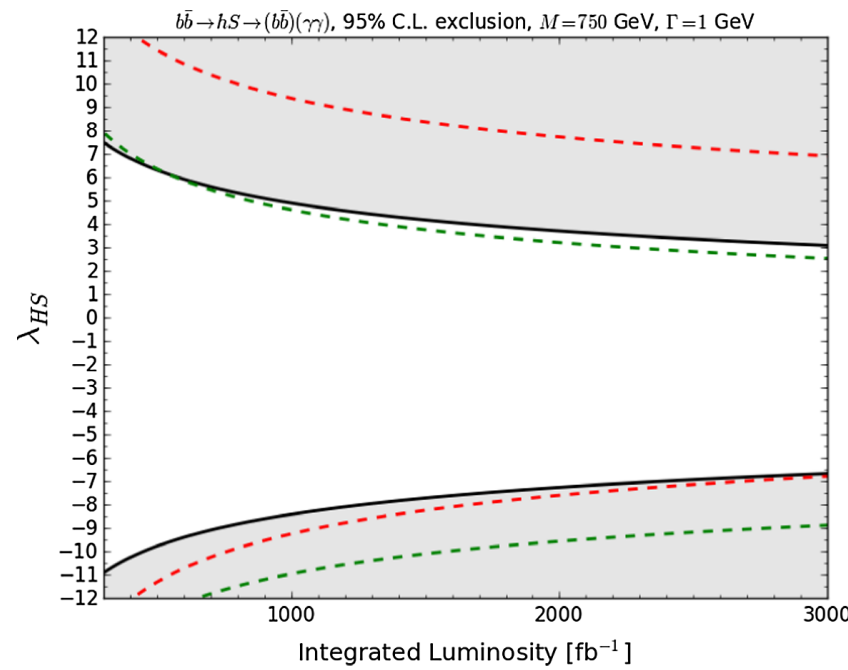

FIG. 19. The grey-shaded area shows the $95 \%$ confidence-level exclusion region for the portal coupling $\lambda_{H S}$ for the $b \bar{b}$-induced case and $M=750 \mathrm{GeV}, \Gamma=1 \mathrm{GeV}$, for the combination of the TBD (red) and OS $\gamma \gamma$ (green) regions as defined in the main text. We have assumed that the single production cross section $\sigma(p p \rightarrow S \rightarrow \gamma \gamma)=5 \mathrm{fb}$.

The lower bound on $\lambda_{H S}$ for the $q \bar{q}$-initiated cases is driven by the TBD region. This is understood by the fact that the TBD region always vanishes near $\lambda_{H S} \sim 0$, as it is dominated by the diagram with an on-shell $s$-channel $S$, making the exclusion region resulting from it symmetric with respect to $\lambda_{H S} \sim 0$, whereas the OS $\gamma \gamma$ region possesses a symmetry point somewhere in $\lambda_{H S}<0$.

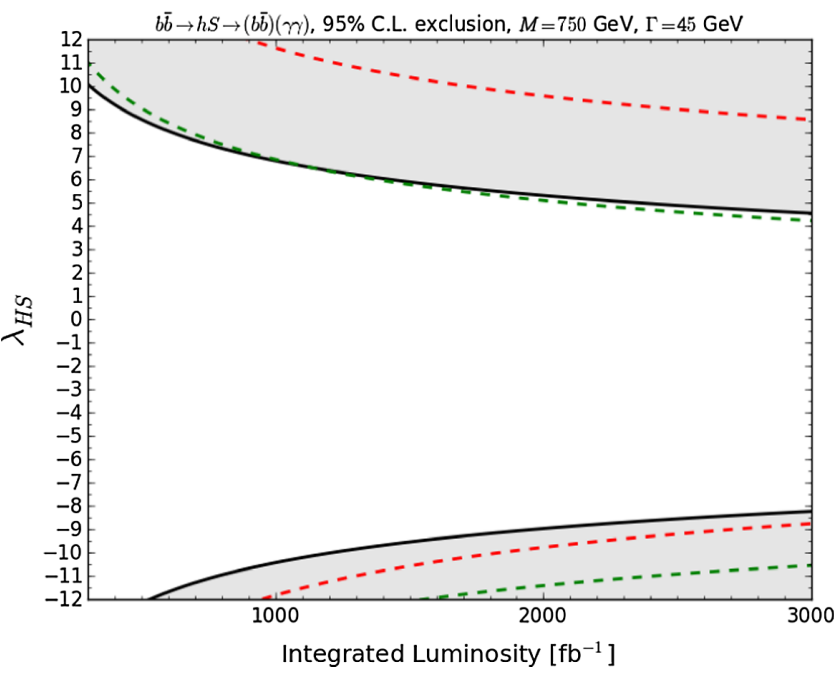

FIG. 20. The grey-shaded area shows the $95 \%$ confidence-level exclusion region for the portal coupling $\lambda_{H S}$ for the $b \bar{b}$-induced case and $M=750 \mathrm{GeV}, \Gamma=45 \mathrm{GeV}$, for the combination of the TBD (red) and OS $\gamma \gamma$ (green) regions as defined in the main text. We have assumed that the single production cross section $\sigma(p p \rightarrow S \rightarrow \gamma \gamma)=5 \mathrm{fb}$.

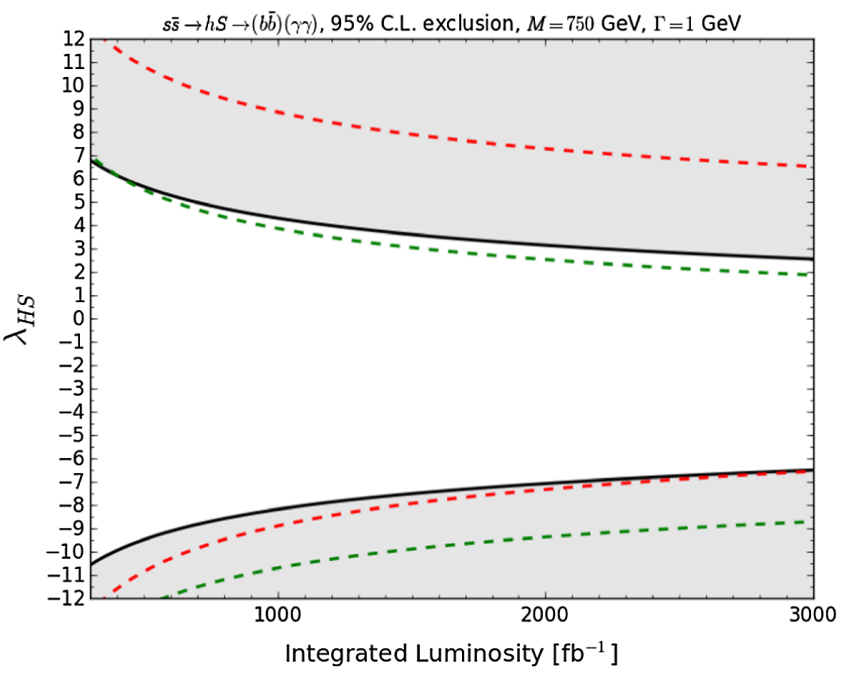

FIG. 21. The grey-shaded area shows the $95 \%$ confidence-level exclusion region for the portal coupling $\lambda_{H S}$ for the $s \bar{s}$-induced case and $M=750 \mathrm{GeV}, \Gamma=1 \mathrm{GeV}$, for the combination of the TBD (red) and OS $\gamma \gamma$ (green) regions as defined in the main text. We have assumed that the single production cross section $\sigma(p p \rightarrow S \rightarrow \gamma \gamma)=5 \mathrm{fb}$.

\section{A. Mixed production of the diphoton resonance}

So far we have investigated production of the scalar resonance initiated purely via either gluon fusion or $q \bar{q}$ annihilation. We can generalize this to "mixed" production via $g g$ and $q \bar{q}$ initial states simultaneously. We concentrate on the scenario of $g g$ and $b \bar{b}$ for simplicity, with the extension to additional quark flavors being straightforward. In that case, the ratio of cross sections, $\rho_{\text {mixed }}$, defined

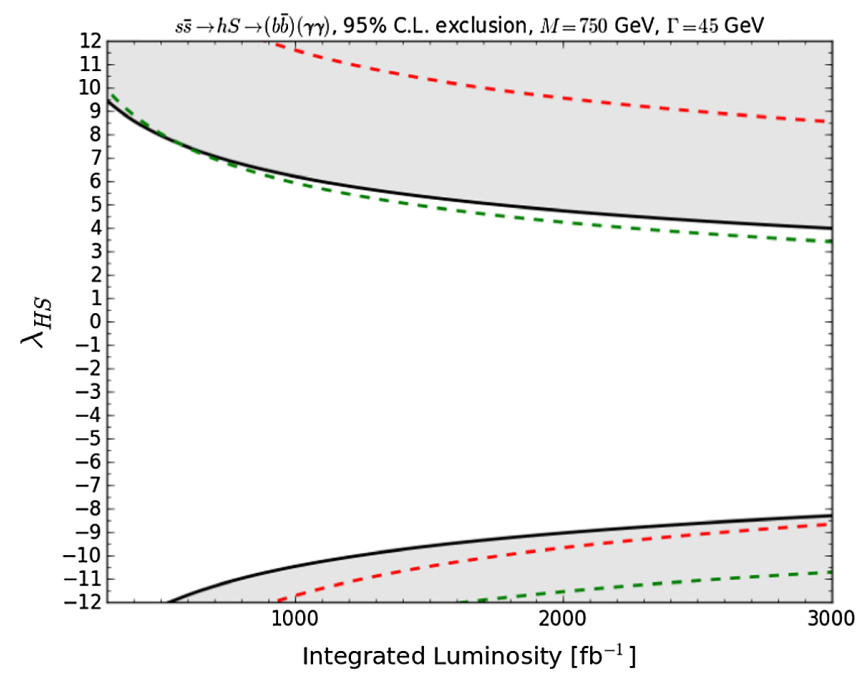

FIG. 22. The grey-shaded area shows the $95 \%$ confidence-level exclusion region for the portal coupling $\lambda_{H S}$ for the $s \bar{s}$-induced case and $M=750 \mathrm{GeV}, \Gamma=45 \mathrm{GeV}$, for the combination of the TBD (red) and OS $\gamma \gamma$ (green) regions as defined in the main text. We have assumed that the single production cross section $\sigma(p p \rightarrow S \rightarrow \gamma \gamma)=5 \mathrm{fb}$. 


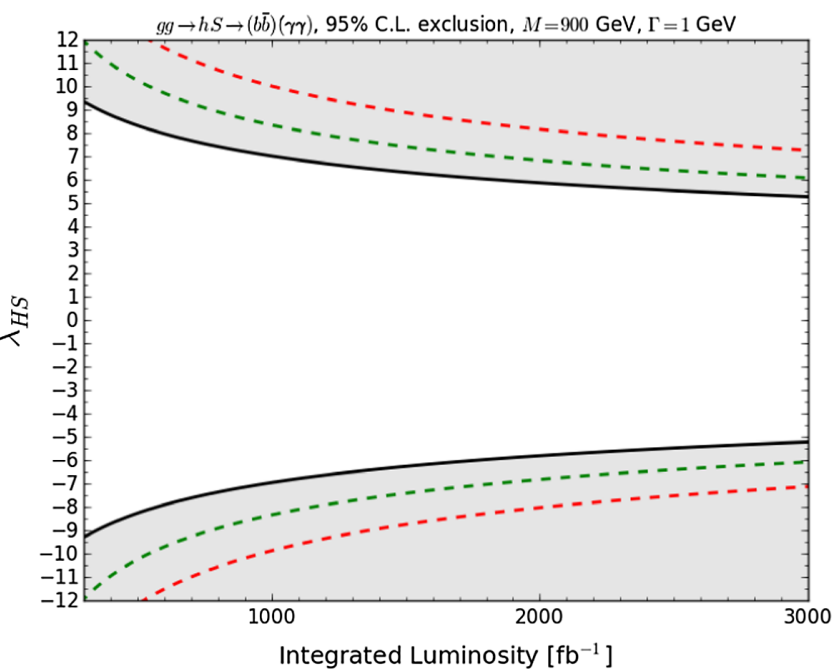

FIG. 23. The grey-shaded area shows the $95 \%$ confidence-level exclusion region for the portal coupling $\lambda_{H S}$ for the $g g$-induced case and $M=900 \mathrm{GeV}, \Gamma=1 \mathrm{GeV}$, for the combination of the TBD (red) and OS $\gamma \gamma$ (green) regions as defined in the main text. We have assumed that the single production cross section $\sigma(p p \rightarrow S \rightarrow \gamma \gamma)=1 \mathrm{fb}$.

between the associated production and single production modes can be written as

$$
\begin{aligned}
\rho_{\text {mixed }} & =\frac{\sigma(b \bar{b} \rightarrow h S \rightarrow h \gamma \gamma)+\sigma(g g \rightarrow h S \rightarrow h \gamma \gamma)}{\sigma(b \bar{b} \rightarrow S \rightarrow \gamma \gamma)+\sigma(g g \rightarrow S \rightarrow \gamma \gamma)} \\
& =\frac{B_{2}\left(\lambda_{H S}\right)\left[\left(y_{d}^{S}\right)^{33}\right]^{2}+G_{2}\left(\lambda_{H S}\right)\left(c_{G}^{S}\right)^{2}}{B_{1}\left[\left(y_{d}^{S}\right)^{33}\right]^{2}+G_{1}\left(c_{G}^{S}\right)^{2}},
\end{aligned}
$$

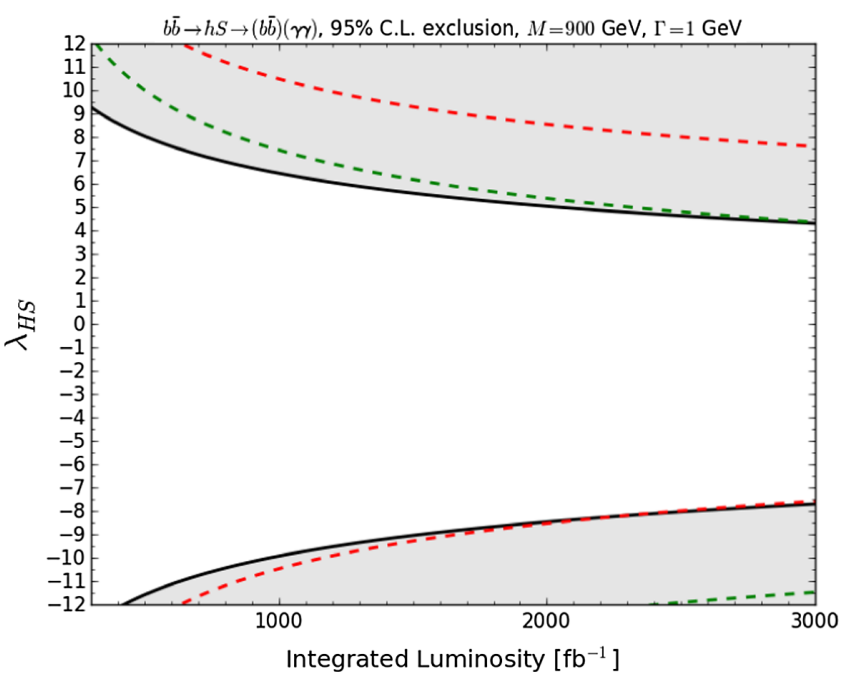

FIG. 24. The grey-shaded area shows the $95 \%$ confidence-level exclusion region for the portal coupling $\lambda_{H S}$ for the $b \bar{b}$-induced case and $M=900 \mathrm{GeV}, \Gamma=1 \mathrm{GeV}$, for the combination of the TBD (red) and OS $\gamma \gamma$ (green) regions as defined in the main text. We have assumed that the single production cross section $\sigma(p p \rightarrow S \rightarrow \gamma \gamma)=1 \mathrm{fb}$.

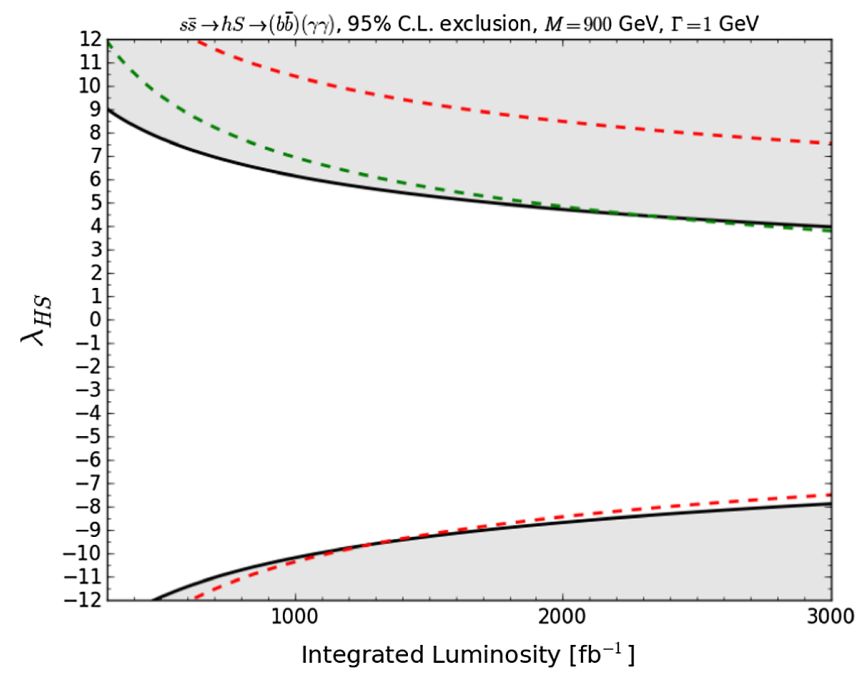

FIG. 25. The grey-shaded area shows the $95 \%$ confidence-level exclusion region for the portal coupling $\lambda_{H S}$ for the $s \bar{s}$-induced case and $M=900 \mathrm{GeV}, \Gamma=1 \mathrm{GeV}$, for the combination of the TBD (red) and OS $\gamma \gamma$ (green) regions as defined in the main text. We have assumed that the single production cross section $\sigma(p p \rightarrow S \rightarrow \gamma \gamma)=1 \mathrm{fb}$.

where $B_{2}\left(\lambda_{H S}\right), G_{2}\left(\lambda_{H S}\right)$ are functions of the portal coupling $\lambda_{H S}$ and $B_{1}, G_{1}$ are constants with respect to the portal coupling, all to be determined. Defining $\theta \equiv\left|\left[\left(y_{d}^{S}\right)^{33}\right] / c_{G}^{S}\right|$, the above expression can be rewritten as

$$
\rho_{\text {mixed }}=\frac{B_{2}\left(\lambda_{H S}\right) \theta^{2}+G_{2}\left(\lambda_{H S}\right)}{B_{1} \theta^{2}+G_{1}} .
$$

By considering the limits $\theta \rightarrow 0$ and $\theta \rightarrow \infty$, and dividing the numerator and denominator by $G_{1}$, we can deduce that

$$
\rho_{\text {mixed }}=\frac{\rho\left(b \bar{b}, \lambda_{H S}\right) r_{b g} \theta^{2}+\rho\left(g g, \lambda_{H S}\right)}{r_{b g} \theta^{2}+1},
$$

where $\rho\left(b \bar{b}, \lambda_{H S}\right)$ and $\rho\left(g g, \lambda_{H S}\right)$ are the ratios of cross sections as functions of the portal coupling as defined in Eq. (7), for the cases of pure production initiated via either $b \bar{b}$ or $g g$, respectively, and $r_{b g}$ is the ratio of pure single production cross sections for $\theta=1: r_{b g}=B_{1} / G_{1}$. The former two functions have already been determined in the analysis of the pure cases. The constant $r_{b g}$ was calculated explicitly via Monte Carlo simulation to be $r_{b g} \approx$ $1.02,0.69,0.49$ for $M=600,750,900 \mathrm{GeV}$, respectively. The values are approximately equal for both the narrow width case $(\Gamma=1 \mathrm{GeV})$ and the larger width case $(\Gamma=45 \mathrm{GeV})$.

Using Eq. (11), we can deduce an expression for the predicted number of signal events after the application of analysis cuts in the mixed production case 


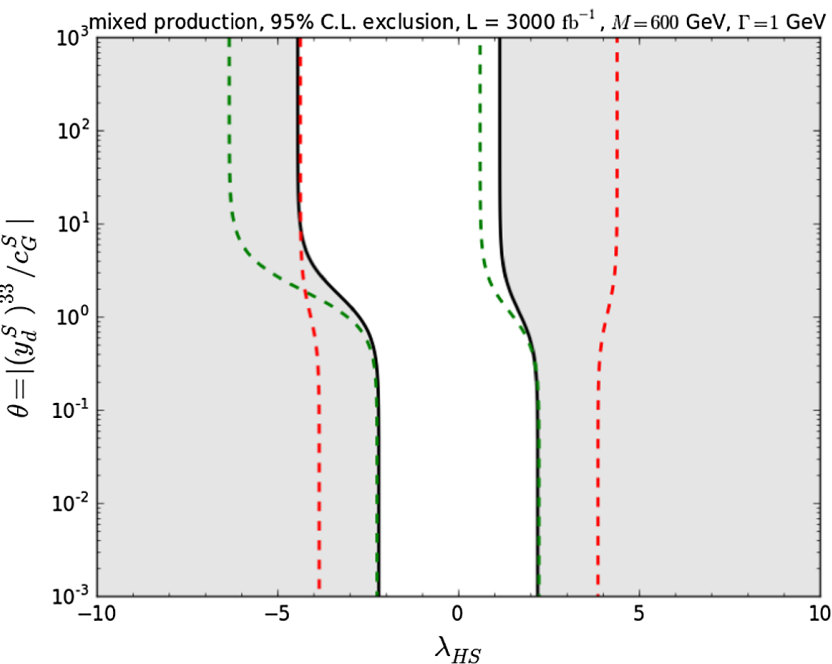

FIG. 26. The 95\% confidence-level exclusion region for the ratio of couplings to $b \bar{b}$ over the coupling to the $g g$ initial states, $\theta=\left[\left(y_{d}^{S}\right)^{33}\right] / c_{G}^{S}$, versus the portal coupling $\lambda_{H S}$ for $M=600 \mathrm{GeV}, \Gamma=1 \mathrm{GeV}$. The excluded region coming from the combination of the TBD (red) and OS $\gamma \gamma$ (green) regions is grey shaded. We have assumed that the single production cross section $\sigma(p p \rightarrow S \rightarrow \gamma \gamma)=10 \mathrm{fb}$

$N_{\text {mixed }}=\frac{r_{b g} \theta^{2}}{r_{b g} \theta^{2}+1} N\left(b \bar{b}, \lambda_{H S}\right)+\frac{1}{r_{b g} \theta^{2}+1} N\left(g g, \lambda_{H S}\right)$,

where $N\left(b \bar{b}, \lambda_{H S}\right)$ and $N\left(g g, \lambda_{H S}\right)$ are the expected signal events for either pure $b \bar{b}$ or pure $g g$ production for a given value of $\lambda_{H S}$, at a specific integrated luminosity.

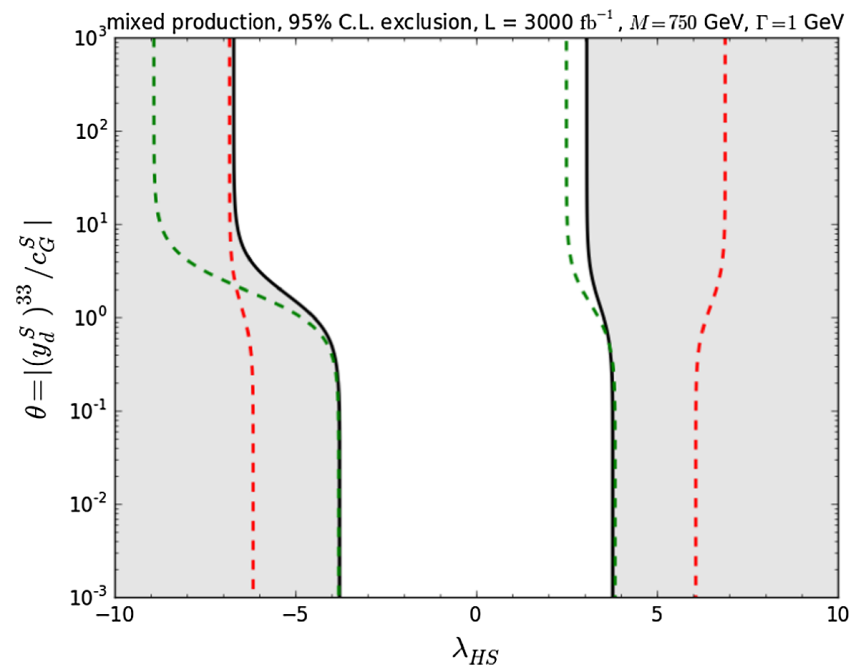

FIG. 27. The 95\% confidence-level exclusion region for the ratio of couplings to $b \bar{b}$ over the coupling to the $g g$ initial states, $\theta=\left[\left(y_{d}^{S}\right)^{33}\right] / c_{G}^{S}$, versus the portal coupling $\lambda_{H S}$ for $M=750 \mathrm{GeV}, \Gamma=1 \mathrm{GeV}$. The excluded region coming from the combination of the TBD (red) and OS $\gamma \gamma$ (green) regions is grey shaded. We have assumed that the single production cross section $\sigma(p p \rightarrow S \rightarrow \gamma \gamma)=5 \mathrm{fb}$.

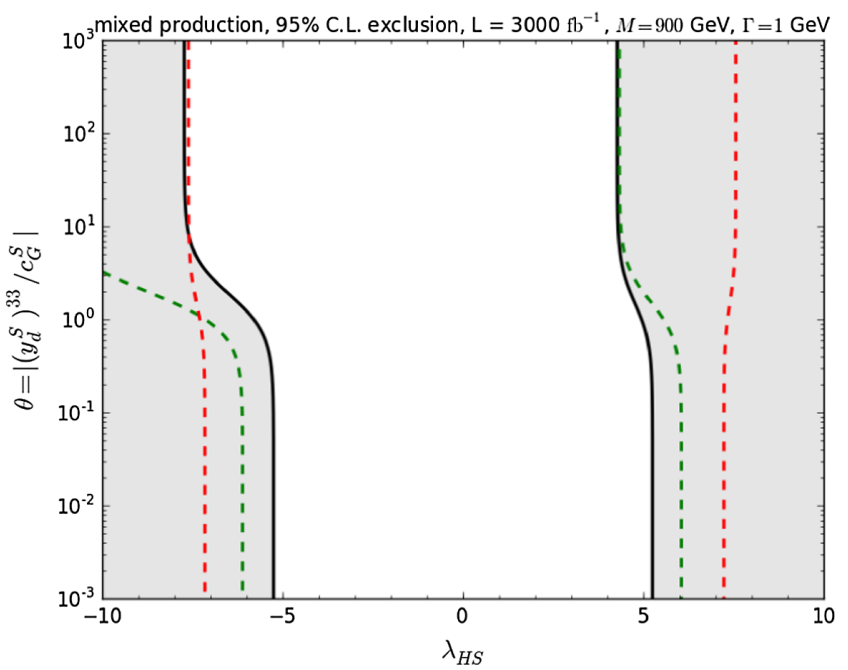

FIG. 28. The 95\% confidence-level exclusion region for the ratio of couplings to $b \bar{b}$ over the coupling to the $g g$ initial states, $\theta=\left[\left(y_{d}^{S}\right)^{33}\right] / c_{G}^{S}$, versus the portal coupling $\lambda_{H S}$ for $M=900 \mathrm{GeV}, \Gamma=1 \mathrm{GeV}$. The excluded region coming from the combination of the TBD (red) and OS $\gamma \gamma$ (green) regions is grey shaded. We have assumed that the single production cross section $\sigma(p p \rightarrow S \rightarrow \gamma \gamma)=1 \mathrm{fb}$.

The predicted number of background events after a given set of analysis cuts is constant with $\theta$, apart from the associated production of the diphoton resonance with a $b \bar{b}$ pair, which was found to be significant only in the $g g$ initiated scenario. This background scales as $N_{\text {assoc }}=$ $N_{\text {assoc }, 0} /\left(r_{b g} \theta^{2}+1\right)$, where $N_{\text {assoc }, 0}$ is the expected number

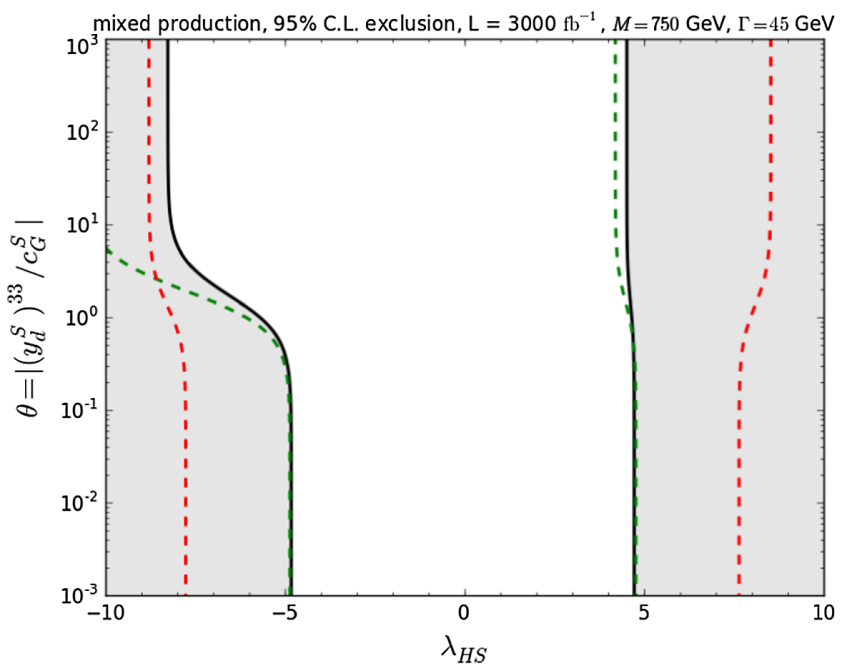

FIG. 29. The 95\% confidence-level exclusion region for the ratio of couplings to $b \bar{b}$ over the coupling to the $g g$ initial states, $\theta=\left[\left(y_{d}^{S}\right)^{33}\right] / c_{G}^{S}$, versus the portal coupling $\lambda_{H S}$ for $M=750 \mathrm{GeV}, \Gamma=45 \mathrm{GeV}$. The excluded region coming from the combination of the TBD (red) and OS $\gamma \gamma$ (green) regions is grey shaded. We have assumed that the single production cross section $\sigma(p p \rightarrow S \rightarrow \gamma \gamma)=5 \mathrm{fb}$. 
of events for the $b \bar{b} S$ associated production after analysis cuts in the case of pure $g g$-initiated production.

Using the expression of Eq. (12) and the event numbers for the two analysis regions TBD and OS $\gamma \gamma$ obtained for the pure production modes, we can derive constraints on the $\left(\theta, \lambda_{H S}\right)$ plane. These are shown in Figs. 26-28 and 29, for masses $M=600,750,900 \mathrm{GeV}$ and $\Gamma=1 \mathrm{GeV}$ and for $M=750 \mathrm{GeV}$ and $\Gamma=45 \mathrm{GeV}$, respectively, at an integrated luminosity of $3000 \mathrm{fb}^{-1}$ at $13 \mathrm{TeV}$. It can be seen that in the limits $\theta \rightarrow 0$ and $\theta \gg 1$, corresponding to $g g$ - or $b \bar{b}$-dominated production, respectively, one can recover the pure production constraints of Figs. 14-25 obtained at $3000 \mathrm{fb}^{-1}$.

\section{DISCUSSION AND CONCLUSIONS}

We have investigated the associated production of a diphoton scalar resonance with a Higgs boson and have employed the $p p \rightarrow h S \rightarrow(b \bar{b})(\gamma \gamma)$ final state to obtain constraints on the portal coupling with the SM Higgs boson $\lambda_{H S}$, at the LHC. We have considered three benchmark scalar masses, $M=600,750,900 \mathrm{GeV}$, and we have assumed that the inclusive single production cross section is $\sigma(p p \rightarrow S \rightarrow \gamma \gamma)=10,5,1 \mathrm{fb}$, respectively, compatible with current experimental constraints. To construct expected constraints we considered the null hypothesis (i.e., the supposed "true" underlying theory) to correspond to gluon-fusion-initiated production with vanishing portal coupling, $\lambda_{H S}=0$. We then first analyzed the case of either pure gluon-fusion-induced production or production via quark-antiquark annihilation. For gluon-fusion production one can impose constraints on the portal coupling at the end of the HL-LHC run of $\left|\lambda_{H S}\right| \lesssim 2,4,5$ for $M=600,750,900 \mathrm{GeV}$, respectively, and $\Gamma=1 \mathrm{GeV}$, while $\left|\lambda_{H S}\right| \lesssim 5$ in the case of large width $(\Gamma=45 \mathrm{GeV})$ and $M=750 \mathrm{GeV}$. For quarkantiquark annihilation, the production of an on-shell scalar and the Higgs boson is enhanced by a contact interaction $\sim q \bar{q} h S$, originating from the same $D=5$ operator that mediates single $\mathcal{S}$ production. This implies that the cross section is non-negligible even for vanishing portal coupling $\lambda_{H S}=0$. This fact can be exploited to exclude the whole plane of $\lambda_{H S}$, thus excluding the hypothesized production via $q \bar{q}$ annihilation. For the case of $b \bar{b}$ we find that a region inside $\lambda_{H S} \in$ $[\sim-4, \sim 1],[\sim-7, \sim 3],[\sim-8, \sim 4]$ for $M=600,750$, $900 \mathrm{GeV}$, respectively, could remain unconstrained at the end of the HL-LHC in the narrow width scenario, while $\lambda_{H S} \in[\sim-8, \sim 5]$ for large width and $M=750 \mathrm{GeV}$. For the case of $s \bar{s}$ annihilation we find the same unconstrained regions to good approximation for narrow width, while we obtain $\lambda_{H S} \in[\sim-8, \sim 4]$ in the case of large width, where $M=750 \mathrm{GeV}$. We have also considered "mixed" production via gluon fusion and $b \bar{b}$ annihilation and derived constraints on the plane of ratio of the corresponding couplings versus $\lambda_{H S}$ for an integrated luminosity of $3000 \mathrm{fb}^{-1}$.

Note that since a measurement of $\lambda_{H S}$ can straightforwardly be translated into a measurement of $\mu_{S}$, these numbers suggest that it is possible to exclude, for example, the scenario where the mass of $S$ stems from EWSB $\left(\mu_{S}=0\right)$, which corresponds to $\lambda_{H S}=M^{2} / v^{2} \approx 5.9,9.3$, 13.4 (still assuming linear terms in $\mathcal{S}$ to vanish). ${ }^{12}$ Furthermore, if additional decay modes of the resonance $S$ are discovered beyond $\gamma \gamma$, it is conceivable that a combined analysis in various channels would be able to exclude all possible values of $\lambda_{H S}$-for the case of $q \bar{q}$ production-thus providing an independent method of determining the production mode. Conversely, the analysis of the present article demonstrates that if the production mechanism is constrained via alternative means, it will be possible to obtain meaningful constraints on the interaction of a new scalar resonance and the Higgs boson, allowing determination of its relation to electroweak symmetry breaking.

\section{ACKNOWLEDGMENTS}

We would like to thank Peter Richardson, Jernej Kamenik, José Zurita, Tania Robens, and Eleni Vryonidou for useful comments and discussion. A. C. acknowledges support by a Marie Skłodowska-Curie Individual Fellowship of the European Community's Horizon 2020 Framework Programme for Research and Innovation under Contract No. 659239 (NP4theLHC14). The research of F. G. is supported by a Marie Curie Intra European Fellowship within the 7th European Community Framework Programme (Grant No. PIEF-GA-2013628224). A. P. acknowledges support by the MCnetITN FP7 Marie Curie Initial Training Network PITN-GA-2012315877 and a Marie Curie Intra European Fellowship within the 7th European Community Framework Programme (Grant No. PIEF-GA-2013-622071).

\section{APPENDIX A: THE FULL SET OF OPERATORS}

In this appendix, we provide the full set of operators for the SM in the presence of an additional dynamical scalar singlet $\mathcal{S}$, up to $D=5$. The fact that $\mathcal{S}$ does not transform under the SM gauge group makes the construction straightforward: in the case that it is $C P$ even (with $C P$-conserving interactions), each additional operator will consist of a gauge invariant SM operator, multiplied by powers of $\mathcal{S}$ (and potentially derivatives). ${ }^{13}$

\footnotetext{
${ }^{12}$ This is somewhat similar to the case of testing the presence/ size of the $\mu$ term in the SM Higgs potential in Higgs pair production [71].

${ }^{13}$ We neglect the only $D=5$ operator consisting solely of SM building blocks - the Weinberg operator-since the tiny neutrino masses suggest that it is suppressed by a very large scale $\Lambda \sim M_{\mathrm{GUT}}$
} 
This means in particular that, schematically, $\mathcal{L} \supset \mathcal{L}_{\mathrm{SM}}+$ $\mathcal{L}_{\mathrm{SM}} \cdot \mathcal{S}$. If all operators in the $\mathrm{SM}$ would be marginal, i.e., feature $D=4$, this would already correspond to the full Lagrangian at $D \leq 5$, connecting the SM with the new resonance. In turn, the only operators that are missing (up to pure NP terms) are those containing the single relevant (i.e., $D<4$ ) operator of the SM, $|H|^{2}$. This can be multiplied by up to three powers of $\mathcal{S}$ as well as by $\partial^{2} \mathcal{S}$ (the latter being equivalent via integration by parts to $\mathcal{S} \partial^{2}|H|^{2}$ ). This finally leads to the Lagrangian ${ }^{14}$

$$
\begin{aligned}
\mathcal{L}_{\mathrm{eff}}= & \mathcal{L}_{\mathrm{SM}}+\frac{1}{2} \partial_{\mu} \mathcal{S} \partial^{\mu} \mathcal{S}-\frac{1}{2} \mu_{S}^{2} \mathcal{S}^{2} \\
& -\frac{\lambda_{S}^{\prime}}{2 \sqrt{2}} v \mathcal{S}^{3}-\frac{\lambda_{S}}{4} \mathcal{S}^{4}-\lambda_{H S}^{\prime} v|H|^{2} \mathcal{S}-\lambda_{H S}|H|^{2} \mathcal{S}^{2} \\
& -\frac{\mathcal{S}}{\Lambda}\left[c_{\lambda S} \mathcal{S}^{4}+c_{H S}|H|^{2} \mathcal{S}^{2}+c_{\lambda H}|H|^{4}\right] \\
& -\left(y_{d}^{S}\right)^{i j} \frac{\mathcal{S}}{\Lambda} \bar{Q}_{L}^{i} H d_{R}^{j}-\left(y_{u}^{S}\right)^{i j} \frac{\mathcal{S}}{\Lambda} \bar{Q}_{L}^{i} \tilde{H} u_{R}^{j}+\text { H.c. } \\
& -\frac{\mathcal{S}}{\Lambda} \frac{1}{16 \pi^{2}}\left[g^{\prime 2} c_{B}^{S} B_{\mu \nu} B^{\mu \nu}+g^{2} c_{W}^{S} W^{I \mu \nu} W_{\mu \nu}^{I}\right. \\
& \left.+g_{S}^{2} c_{G}^{S} G^{a \mu \nu} G_{\mu \nu}^{a}\right] .
\end{aligned}
$$

Note that we have used equations of motion/field redefinitions to eliminate the operators $\mathcal{S} / \Lambda\left(\partial^{\mu} \mathcal{S}\right)^{2}$, $\mathcal{S} / \Lambda\left|D_{\mu} H\right|^{2}, \mathcal{S} / \Lambda \partial^{2}|H|^{2}$ and those of type $\mathcal{S} / \Lambda \bar{q} D_{\mu} \gamma^{\mu} q$. The couplings in the third line of (A1) and $\lambda_{S, H S}^{\prime}$, which are those that have been neglected in (1), do not contribute to the process under consideration to good approximation: They either do not enter at leading order, or (in the case of the $|H|^{2,4} \mathcal{S}$ interactions) at most contribute to a diagram with a (strongly suppressed) offshell Higgs boson propagator, discussed in Appendix B. The same holds true for the $S^{4}$ term, which was kept for the discussion in Sec. I.

\section{APPENDIX B: FITS FOR THE RATIO $\rho$ INCLUDING A LINEAR TERM}

In addition to the diagrams of Figs. 1 and 2, there can be contributions to the final state under consideration originating from $h h S$ interactions (for example, an intermediate $s$-channel Higgs boson to $h S$ or an $S \rightarrow h h$ intermediate state), provided the following triple scalar interaction, linear in $S$, is nonvanishing,

\footnotetext{
${ }^{14}$ See also $[40,72]$. We do not include leptons, as they do not enter the examined processes to the order considered. Moreover, note that (induced) tadpoles in $\mathcal{S}$ can always be removed via a field redefinition.
}

TABLE V. The $\rho$ fit coefficients for the general case of including an interaction term proportional to $\mathcal{L} \supset-\kappa_{H S} v h^{2} S$ for width $\Gamma=1 \mathrm{GeV}$ and $M=750 \mathrm{GeV}$.

\begin{tabular}{ccrr}
\hline \hline$i$ & $\delta_{i}^{b}$ & \multicolumn{1}{c}{$\delta_{i}^{s}$} & \multicolumn{1}{c}{$\delta_{i}^{g}$} \\
\hline 1 & $8.27 \times 10^{-3}$ & $1.05 \times 10^{-2}$ & $<\mathcal{O}\left(10^{-5}\right)$ \\
2 & $1.93 \times 10^{-4}$ & $-2.30 \times 10^{-4}$ & $\mathcal{O}\left(10^{-7}\right)$ \\
3 & $8.26 \times 10^{-4}$ & $2.54 \times 10^{-5}$ & $<\mathcal{O}\left(10^{-6}\right)$ \\
4 & $3.08 \times 10^{-3}$ & $3.71 \times 10^{-3}$ & $<\mathcal{O}\left(10^{-6}\right)$ \\
5 & $2.40 \times 10^{-5}$ & $6.00 \times 10^{-5}$ & $<\mathcal{O}\left(10^{-8}\right)$ \\
6 & $8.60 \times 10^{-4}$ & $9.27 \times 10^{-4}$ & $1.33 \times 10^{-3}$ \\
\hline \hline
\end{tabular}

$$
-\kappa_{H S} v h^{2} S \subset-\lambda_{H S}^{\prime} v|H|^{2} \mathcal{S}-c_{\lambda H} \frac{\mathcal{S}}{\Lambda}|H|^{4},
$$

with $\kappa_{H S}=1 / 2 \lambda_{H S}^{\prime}+3 /(2 \Lambda) c_{\lambda H}$.

In this scenario, terms will appear in the cross section ratio proportional to $\kappa_{H S}$ and $\kappa_{H S}^{2}$. Because of interference with the new $h h S$ diagrams (note that also the "non-signal" $S \rightarrow h h$ interferes with the signal diagrams after an offshell $h \rightarrow \gamma \gamma$ ), some dependence on the coupling of the resonance $S$ to photons, $c_{\gamma}^{S}$, as well as the production couplings, $\left(y_{d}^{S}\right)^{22},\left(y_{d}^{S}\right)^{33}$, and $c_{G}^{S}$, is introduced in the denominator of the cross section ratio. Because of this, smaller values of $\left|c_{\gamma}^{S}\right|$ and the production couplings enhance the effect of the new contributions proportional to $\kappa_{H S}$ and $\kappa_{H S}^{2}$. To take these effects into account and at the same time remain conservative, we set $\left|c_{\gamma}^{S}\right|$ to the possible minimal value that produces a $\sigma(p p \rightarrow S \rightarrow \gamma \gamma) \sim 5 \mathrm{fb}$ for $M=750 \mathrm{GeV}$, as derived in Ref. [24]. This leads to $\left|c_{\gamma}^{S}\right| \gtrsim\{60,100,10\}$ for $s \bar{s}, b \bar{b}$, and $g g$ production, respectively, where here and in the following we assume a normalization of $\Lambda=1 \mathrm{TeV}$. Moreover, one can derive conservative lower bounds on $\left|\left(y_{d}^{S}\right)^{22}\right|,\left|\left(y_{d}^{S}\right)^{33}\right|$, and $\left|c_{g}^{S}\right|$, by demanding $\sigma(p p \rightarrow S) \gtrsim 5 \mathrm{fb}$, leading to $\left|\left(y_{d}^{S}\right)^{22}\right| \gtrsim 0.15$, $\left|\left(y_{d}^{S}\right)^{33}\right| \gtrsim 0.2$, and $\left|c_{g}^{S}\right| \gtrsim 0.25$. We set these to their minimal values as well in what follows.

We can then parametrize the ratio $\rho\left(x x^{\prime}\right)=\sigma\left(x x^{\prime} \rightarrow h \gamma \gamma\right) /$ $\sigma\left(x x^{\prime} \rightarrow S \rightarrow \gamma \gamma\right), x x^{\prime}=b \bar{b}, s \bar{s}, g g$, by ${ }^{15}$

$$
\begin{aligned}
\rho\left(x x^{\prime}\right)= & \delta_{1}^{x}+\delta_{2}^{x} \kappa_{H S} \lambda_{H S}+\delta_{3}^{x} \kappa_{H S} \\
& +\delta_{4}^{x} \lambda_{H S}+\delta_{5}^{x} \kappa_{H S}^{2}+\delta_{6}^{x} \lambda_{H S}^{2},
\end{aligned}
$$

where $\delta_{i}^{b, s, g}$ are coefficients to be determined.

An estimate of the coefficients in this conservative scenario, obtained numerically, is given in Table $\mathrm{V}$ for width $\Gamma=1 \mathrm{GeV}$ and $M=750 \mathrm{GeV}$. The contributions from diagrams with an off-shell Higgs boson are a priori not fully negligible for the $b \bar{b}$-induced case, simply

\footnotetext{
${ }^{15}$ For the case of vanishing $\kappa_{H S}$, this more general definition coincides with Eq. (7) to good approximation.
} 
because of interference of the $s$-channel $h \rightarrow h S$ with the large matrix elements with the on-shell $S$ in the final state.

To investigate the size of $\kappa_{H S}$ that renders the off-shell Higgs boson contributions significant to the $p p \rightarrow h \gamma \gamma$ process, we consider the ratio $\rho\left(\kappa_{H S}, \lambda_{H S}\right) / \rho\left(\kappa_{H S}=\right.$ $\left.0, \lambda_{H S}\right)=1+r$, where $r$ is a number that characterizes the fractional change in the ratio $\rho$ due to $\kappa_{H S}$ for a given value of $\lambda_{H S}$. If we choose $r=1$, which implies $\mathcal{O}(1)$ changes due to $\kappa_{H S}$, and solve for $\kappa_{H S}$ for values $\lambda_{H S} \sim$ $\mathcal{O}(1)$ we obtain $\left|\kappa_{H S}\right| \sim\left\{\mathcal{O}(10), \mathcal{O}(10), \mathcal{O}\left(10^{2}\right)\right\}$ for $s \bar{s}$, $b \bar{b}$, and $g g$ production, respectively.

Since the gauge-invariant terms in the Lagrangian generating this coupling also induce a Higgs-scalar mixing, they cannot be arbitrarily large. Indeed, Higgs data can constrain $\left|\kappa_{H S}\right| \lesssim 4$ at $95 \%$ confidence level [73-75]. ${ }^{16}$ Therefore, given the values calculated in the previous paragraph, for $\kappa_{H S}$ to eventually have a non-negligible effect on the analysis of the present article, this bound needs to be violated, and setting $\kappa_{H S}=0$ is a justified approximation. Despite the fact that we focused on $M=750 \mathrm{GeV}$, the analysis is expected to give similar estimates for the other benchmark points considered in the main analysis of this article, $M=600,900 \mathrm{GeV}$.

\footnotetext{
${ }^{16}$ Here, we have neglected the impact of the second operator in (B1), $\mathcal{S}|H|^{4}$, which breaks the correlation between Higgs-scalar mixing and the $h^{2} S$ interaction.
}

[1] A. Falkowski, C. Gross, and O. Lebedev, J. High Energy Phys. 05 (2015) 057.

[2] B. Bellazzini, C. Cski, and J. Serra, Eur. Phys. J. C 74, 2766 (2014).

[3] B. Bellazzini, R. Franceschini, F. Sala, and J. Serra, J. High Energy Phys. 04 (2016) 072.

[4] U. Ellwanger, C. Hugonie, and A. M. Teixeira, Phys. Rep. 496, 1 (2010).

[5] R. Hempfling, Phys. Lett. B 379, 153 (1996).

[6] C. Englert, J. Jaeckel, V. V. Khoze, and M. Spannowsky, J. High Energy Phys. 04 (2013) 060.

[7] T. Gherghetta, N. Nagata, and M. Shifman, Phys. Rev. D 93, 115010 (2016).

[8] K. Tsumura and L. Velasco-Sevilla, Phys. Rev. D 81, 036012 (2010).

[9] M. Bauer, T. Schell, and T. Plehn, Phys. Rev. D 94, 056003 (2016).

[10] V. Khachatryan et al. (CMS Collaboration), Eur. Phys. J. C 74, 3076 (2014).

[11] G. Aad et al. (ATLAS Collaboration), Phys. Rev. D 90, 112015 (2014).

[12] CERN Technical Report No. ATLAS-CONF-2015-081, 2015.

[13] CERN Technical Report No. ATLAS-CONF-2016-018, 2016.

[14] CERN Technical Report No. CMS-PAS-EXO-15-004, 2015.

[15] CERN Technical Report No. CMS-PAS-EXO-16-018, 2016.

[16] CERN Technical Report No. ATLAS-CONF-2016-059, 2016.

[17] V. Khachatryan et al. (CMS Collaboration), Phys. Lett. B 767, 147 (2017).

[18] R. Franceschini, G. F. Giudice, J. F. Kamenik, M. McCullough, A. Pomarol, R. Rattazzi, M. Redi, F. Riva, A. Strumia, and R. Torre, J. High Energy Phys. 03 (2016) 144.
[19] A. Angelescu, A. Djouadi, and G. Moreau, Phys. Lett. B 756, 126 (2016).

[20] S. Knapen, T. Melia, M. Papucci, and K. Zurek, Phys. Rev. D 93, 075020 (2016).

[21] S. Di Chiara, L. Marzola, and M. Raidal, Phys. Rev. D 93, 095018 (2016).

[22] S. D. McDermott, P. Meade, and H. Ramani, Phys. Lett. B 755, 353 (2016).

[23] J. Ellis, S. A. R. Ellis, J. Quevillon, V. Sanz, and T. You, J. High Energy Phys. 03 (2016) 176.

[24] R. S. Gupta, S. Jäger, Y. Kats, G. Perez, and E. Stamou, J. High Energy Phys. 07 (2016) 145.

[25] A. Falkowski, O. Slone, and T. Volansky, J. High Energy Phys. 02 (2016) 152.

[26] A. Alves, A. G. Dias, and K. Sinha, Phys. Lett. B 757, 39 (2016).

[27] F. Goertz, J. F. Kamenik, A. Katz, and M. Nardecchia, J. High Energy Phys. 05 (2016) 187.

[28] M. Son and A. Urbano, J. High Energy Phys. 05 (2016) 181.

[29] J. Gao, H. Zhang, and H. X. Zhu, Eur. Phys. J. C 76, 348 (2016)

[30] A. Salvio and A. Mazumdar, Phys. Lett. B 755, 469 (2016).

[31] J. Gu and Z. Liu, Phys. Rev. D 93, 075006 (2016).

[32] A. Djouadi, J. Ellis, R. Godbole, and J. Quevillon, J. High Energy Phys. 03 (2016) 205.

[33] A. Salvio, F. Staub, A. Strumia, and A. Urbano, J. High Energy Phys. 03 (2016) 214.

[34] C. Gross, O. Lebedev, and J. M. No, arXiv:1602.03877.

[35] F. Goertz, A. Katz, M. Son, and A. Urbano, J. High Energy Phys. 07 (2016) 136.

[36] J. Bernon, A. Goudelis, S. Kraml, K. Mawatari, and D. Sengupta, J. High Energy Phys. 05 (2016) 128.

[37] G. Panico, L. Vecchi, and A. Wulzer, J. High Energy Phys. 06 (2016) 184.

[38] J. F. Kamenik, B. R. Safdi, Y. Soreq, and J. Zupan, J. High Energy Phys. 07 (2016) 042. 
[39] M. Chala, C. Grojean, M. Riembau, and T. Vantalon, Phys. Lett. B 760, 220 (2016).

[40] R. Franceschini, G. F. Giudice, J. F. Kamenik, M. McCullough, F. Riva, A. Strumia, and R. Torre, J. High Energy Phys. 07 (2016) 150.

[41] D. Buttazzo, A. Greljo, and D. Marzocca, Eur. Phys. J. C 76, 116 (2016).

[42] L. Berthier, J. M. Cline, W. Shepherd, and M. Trott, J. High Energy Phys. 04 (2016) 084.

[43] P. S. B. Dev, R. N. Mohapatra, and Y. Zhang, J. High Energy Phys. 02 (2016) 186.

[44] P. Roig and J. J. Sanz-Cillero, Phys. Rev. D 94, 095021 (2016).

[45] S. Dawson and I. M. Lewis, Phys. Rev. D 95, 015004 (2017)

[46] J. Zhang and S. Zhou, Chin. Phys. C 40, 081001 (2016).

[47] F. Goertz, A. Papaefstathiou, L. L. Yang, and J. Zurita, J. High Energy Phys. 06 (2013) 016.

[48] P. Motylinski, L. Harland-Lang, A. D. Martin, and R. S. Thorne, Nucl. Part. Phys. Proc. 273-275, 2136 (2016).

[49] N. D. Christensen and C. Duhr, Comput. Phys. Commun. 180, 1614 (2009).

[50] A. Alloul, N. D. Christensen, C. Degrande, C. Duhr, and B. Fuks, Comput. Phys. Commun. 185, 2250 (2014).

[51] C. Degrande, C. Duhr, B. Fuks, D. Grellscheid, O. Mattelaer, and T. Reiter, Comput. Phys. Commun. 183, 1201 (2012).

[52] J. Alwall, M. Herquet, F. Maltoni, O. Mattelaer, and T. Stelzer, J. High Energy Phys. 06 (2011) 128.

[53] J. Alwall, R. Frederix, S. Frixione, V. Hirschi, F. Maltoni, O. Mattelaer, H.-S. Shao, T. Stelzer, P. Torrielli, and M. Zaro, J. High Energy Phys. 07 (2014) 079.

[54] M. Bahr et al., Eur. Phys. J. C 58, 639 (2008).

[55] S. Gieseke et al., arXiv:1102.1672.

[56] K. Arnold et al., arXiv:1205.4902.

[57] J. Bellm et al., arXiv:1310.6877.
[58] J. Bellm et al., Eur. Phys. J. C 76, 196 (2016).

[59] V. Hirschi and O. Mattelaer, J. High Energy Phys. 10 (2015) 146.

[60] The ATLAS collaboration, Technical Report No. ATLPHYS-PUB-2013-004, 2013.

[61] The ATLAS collaboration, Technical Report No. ATLPHYS-PUB-2013-009, 2013.

[62] M. Cacciari, G. P. Salam, and G. Soyez, Eur. Phys. J. C 72, 1896 (2012).

[63] M. Cacciari and G. P. Salam, Phys. Lett. B 641, 57 (2006).

[64] U. Baur, T. Plehn, and D. L. Rainwater, Phys. Rev. D 69, 053004 (2004).

[65] J. Baglio, A. Djouadi, R. Grober, M. M. Muhlleitner, J. Quevillon, and M. Spira, J. High Energy Phys. 04 (2013) 151.

[66] W. Yao, arXiv:1308.6302.

[67] V. Barger, L. L. Everett, C. B. Jackson, and G. Shaughnessy, Phys. Lett. B 728, 433 (2014).

[68] N. Chen, C. Du, Y. Fang, and L.-C. Lü, Phys. Rev. D 89, 115006 (2014).

[69] A. Papaefstathiou, K. Sakurai, and M. Takeuchi, J. High Energy Phys. 08 (2014) 176.

[70] S. Stouffer, E. Suchman, L. DeVinnery, S. Star, and R.W. J. Williams, The American Soldier: Adjustment During Army Life (Studies in Social Psychology in World War II, Vol. I) (Princeton University Press, Princeton, NJ, 1949).

[71] F. Goertz, Phys. Rev. D 94, 015013 (2016).

[72] B. Gripaios and D. Sutherland, J. High Energy Phys. 08 (2016) 103.

[73] D. Carmi, A. Falkowski, E. Kuflik, T. Volansky, and J. Zupan, J. High Energy Phys. 10 (2012) 196.

[74] K. Cheung, P. Ko, J. S. Lee, and P.-Y. Tseng, J. High Energy Phys. 10 (2015) 057.

[75] M. Bauer, C. Hoerner, and M. Neubert, J. High Energy Phys. 07 (2016) 094. 